\title{
Beyond brane-Higgs regularization: Clarifying the method and model
}

\author{
Andrei Angelescu $\odot,{ }^{1, *}$ Ruifeng Leng $\odot,{ }^{2, \uparrow}$ Grégory Moreau $\odot,{ }^{2, \$}$ and Florian Nortier $\circledast^{2, \S}$ \\ ${ }^{1}$ Department of Physics and Astronomy, University of Nebraska-Lincoln, Lincoln, Nebraska 68588, USA \\ ${ }^{2}$ Laboratoire de Physique Théorique CNRS \& Univ. Paris-Sud, Université Paris-Saclay, \\ 91405 Orsay, France
}

(Received 24 January 2020; accepted 3 April 2020; published 27 April 2020)

\begin{abstract}
The attractive class of higher-dimensional scenarios, based on a brane-localized Higgs boson coupled to bulk fermions, can address both the puzzle of the structure of the flavor space and the gauge hierarchy problem. In this framework, a key question arises due to the possibility of fermion wave function discontinuities at the Higgs boundary: how to build rigorously the Lagrangian and calculate the fermion mass spectrum as well as the effective four-dimensional (4D) Yukawa couplings. We show that the proper treatment, leading to physically consistent solutions, does not rely on any Higgs peak regularization but requires the presence of certain bilinear brane terms. In particular, no profile jump should appear, and the Higgs regularizations turn out to suffer from mathematical discrepancies reflected in two noncommutativities of calculation steps debated in the literature. The introduction of bilinear brane terms can alternatively be replaced by vanishing conditions for probability currents at the considered flat interval boundaries. Indeed, both contribute to the definition of the field geometrical configuration of the model, even in the free case. The bilinear brane terms could allow us to elaborate an ultraviolet origin of the chiral nature of the Standard Model and of its chirality distribution among quarks/leptons. The current conditions are implemented through essential boundary conditions to be contrasted with the natural boundary conditions derived from the action variation. All these theoretical conclusions are confirmed in particular by the converging exact results of the $4 \mathrm{D}$ versus five-dimensional approaches. The analysis is completed by a description of the appropriate energy cutoff procedure in the present context. The new calculation methods presented, implying the independence of excited fermion masses and 4D Yukawa couplings on the "wrong-chirality" Yukawa terms, have impacts on phenomenological results like the relaxing of previously obtained strong bounds on Kaluza-Klein masses induced by flavor changing reactions generated via treelevel exchanges of the Higgs field.
\end{abstract}

DOI: 10.1103/PhysRevD.101.075048

\section{INTRODUCTION}

The paradigm of scenarios with extra spatial dimensions (and the composite Higgs models dual via the AdS/CFT correspondence) represents an alternative to supersymmetry for addressing the deep gauge hierarchy problem of the Standard Model (SM). In particular, the warped dimension scenarios [1] with SM fields in the bulk [2], although relying on a unique fundamental energy scale, allow us to generate the SM fermion mass hierarchy [3] from a simple geometrical picture of fermion profiles

\footnotetext{
*aangelescu2@unl.edu

†ruifeng.leng@u-psud.fr

\#moreau@th.u-psud.fr

${ }^{\S}$ florian.nortier@th.u-psud.fr
}

Published by the American Physical Society under the terms of the Creative Commons Attribution 4.0 International license. Further distribution of this work must maintain attribution to the author(s) and the published article's title, journal citation, and DOI. Funded by SCOAP ${ }^{3}$. (see, e.g., Refs. [4-9]). To realize those two hierarchical features, the Brout-Englert-Higgs scalar field [10,11], providing a mass via the electroweak (EW) symmetry breaking, must be either stuck exactly on the so-called TeVbrane (boundary of the finite extra dimension) ${ }^{1}$ or located in the bulk with a wave function only peaked at the $\mathrm{TeV}$ brane. Branes are hypersurfaces located in an higher-dimensional space and can arise in the framework of string theories as D-branes which are dynamical objects with quantum properties [18,19] (see also Refs. [20,21] for the supergravity limit of string theories). In contrast, in the gaugeHiggs unification models, as described, for instance, in Ref. [22], protecting the Higgs mass down to lower energies, the Higgs field propagates all along the extra dimension together with matter.

\footnotetext{
${ }^{1}$ There exist other phenomenological motivations, like within neutrino mass models, for the Higgs boson to be stuck at the boundary of an interval [12-16] or fermions to propagate in the bulk [17].
} 
Recently, some attention has been paid to the mathematical context of the interaction between Higgs and fermions both propagating along a (warped) extra dimension [23]; it was found that to avoid possible pathological behaviors in the fermion sector constraints on the fermionic field Lagrangian must be imposed. Besides, when including gravity, the description of braneworld models (branelocalized fields or operators) within an effective field theory must be done carefully as discussed in the holographic context of Ref. [24]. The energy-momentum tensor of brane-localized fields (scalar, spinor, and vector) must also satisfy some constraints induced by Einstein's equations [25]. Such theoretical consistency considerations are interesting from the purely theoretical side and are crucial for the clear understanding of higher-dimensional models being now searched and constrained at the LHC exploratory phase.

In the present paper, we discuss the rigorous treatment of the other case of a boundary-localized Higgs scalar field, interacting with bulk quark/leptons propagating in a finite interval, which presents subtleties that deserve to be looked at more deeply. Such a field configuration occurs in the realistic warped models addressing the fermion mass and gauge hierarchy. The case of bulk matter without interactions is also studied.

Let us recall these subtle aspects. First, a question arises about the correct treatment of the specific object that is the Dirac peak entering each Lagrangian term which involves the brane-Higgs boson. Second, this Dirac peak may induce an unusual discontinuity ${ }^{2}$ in the wave function along the extra dimension (at the Higgs boundary where further conditions arise from the Lagrangian variations) for some of the bulk fermions: the so-called jump problem [27,28]. These five-dimensional (5D) aspects have motivated the introduction $[27,28]$ of a process of regularization of the Higgs Dirac peak (smoothing the peak or shifting it from the boundary) in the calculation of Kaluza-Klein (KK) fermion mass spectra and effective four-dimensional (4D) Yukawa couplings. Although there is no profound theoretical reason to apply such a regularization procedure (forcing interaction-free boundary conditions for fermions), nowadays all the theoretical and phenomenological studies of the warped models with brane-Higgs (see, e.g., Refs. [5,29-33]) are relying on this Higgs peak regularization.

In this paper, we first present the mathematical inconsistencies of this regularization procedure used in the literature. Then, instead of regularizing, we develop the rigorous determination of the profiles - taking into account the mathematical nature of the Dirac peak in the Higgs coupling - which leads to bulk fermion wave functions without discontinuities on the considered extra space. We conclude from this whole approach that neither the profile jump nor a particular problem arises when a proper

\footnotetext{
${ }^{2}$ Field jumps may arise in other frameworks [26].
}

mathematical framework is used, so there is in fact no motivation to introduce a brane-Higgs regularization.

As a consequence, we can now interpret two noncommutativities of calculation steps for Higgs production and decay rates [30-32,34] or for fermion masses and 4D Yukawa couplings [35], previously studied in the literature, to be similar effects and confirmations of the mathematical inconsistencies in the Higgs peak regularization. Besides, the debate in the literature about those two noncommutativities is thus closed by the useless nature of this regularization.

The correct methods without regularization, together with their results, are illustrated here in the derivation of the KK fermion mass spectrum - the same ideas apply to the calculation of effective 4D Yukawa couplings. This spectrum calculation is done in a simplified model with a flat extra dimension, with the minimal field content (to write down a Yukawa interaction), and without gauge symmetry. Nevertheless, this toy model already possesses all the key ingredients to study the delicate brane-Higgs aspects. Hence, our conclusions can be directly extended to the realistic warped models with bulk SM matter addressing the fermion flavor and gauge hierarchy.

Several new spectrum calculation methods are proposed and further allow confirmations of the analytical results. Those methods go through the 4D or 5D approaches (one extra dimension case) and are based on the fermion current determination from either the action variations or the equations of motion. We had to generalize the Noether theorem to include brane-localized terms like the Yukawa couplings. Besides, the correct derivation of the standard free fermion mass spectrum (in the absence of Yukawa interactions) turns out to be a useful starting guide in particular for the 4D approach or more generically for a solid comprehension of such higher-dimensional scenarios.

From a historical point of view, the correct method established here arises naturally in the theory of variational calculus as the Lagrangian boundary term (brane-Higgs coupling to fermions) is included in a new boundary condition instead of entering the equations of motion [36] (via a regularization). Furthermore, the present analysis follows the prescription of considering the Dirac delta to be a distribution. By the way, the Dirac peak and distributions were formalized and validated mathematically during the 1940s by Schwartz [37,38] precisely for the purpose of solving consistently physical problems. Hence, today, it should not be avoided to respect the distribution formalism when facing a physical problem involving an object like the Dirac delta, as it occurs in the present higherdimensional context.

The rigorous results obtained for the KK mass spectrum and effective 4D Yukawa couplings are different from the ones derived in general through the Higgs peak regularization, as it is detailed in the present paper. This difference is physical, affecting then phenomenological studies on indirect searches of KK states at high-energy colliders 
(in particular via the Higgs production and flavor changing neutral currents), and analytical (vanishing of the Yukawa coupling with "wrong" fermion chiralities relatively to the SM), which improves the precise theoretical understanding of the higher-dimensional setup with a brane-localized Higgs field.

Furthermore, the correct mass spectrum obtained here allows us to point out the necessity, for bulk fermions (with or without coupling to a brane-localized scalar field), to have certain bilinear brane terms at boundaries which are fermion mass terms from the point of view of the spinorial structure but do not introduce new mass parameters. ${ }^{3}$ Indeed, such terms guarantee the existence of physical solutions (with correct profile normalizations and Hermitian conjugate boundary conditions and satisfying the decoupling limit argument) derived via the least action principle through the variation calculus. Their necessary presence is confirmed by the nontrivial exact matching between the 5D and 4D analytical calculations of the mass spectrum.

At a brane without Yukawa coupling, instead of including such a bilinear term, we find that one can alternatively impose as an essential boundary condition (in contrast with natural boundary conditions coming from the Lagrangian variations) the condition of a fermion current along the extra dimension vanishing at this brane-and exclusively within the 4D approach in case of a brane with localized Yukawa interaction. Indeed, the generic reason for the presence of bilinear brane terms is the consistent and complete geometrical definition of models with a finite extra spatial interval in which fermionic matter is stuck. Notice that the choice between the bilinear brane term presence and the vanishing fermion current condition relies on the UV completion of the model. Indeed, the vanishing fermion current condition permits alternatively the existence of physical solutions.

Therefore, a first possibility is that the UV completion generates bilinear brane terms for the fermions on both boundaries (those with and without localized Yukawa coupling) of the interval. Then, the geometrical interval definition (interval boundaries and vanishing 5D fermion currents at these boundaries) would be completely contained in the action expression. Now, in case the UV completion would not induce bilinear brane terms on both boundaries, such essential boundary conditions should be imposed at the brane(s) without bilinear terms in order to define well the geometrical configuration and to have acceptable physical solutions. We can thus conclude that, whether the geometrical setup is defined exclusively through the action expression (leading to the natural boundary conditions) or (also) via additional essential

\footnotetext{
${ }^{3}$ The potential 4D effective mass involves a dimensionful product of two profile boundary values.
}

boundary conditions depends on the origin of the model at high energies.

In case the UV completion produces bilinear brane terms for the fermions on both boundaries, at low energies, the chiral nature of the SM as well as its field chirality distribution [left-handed $S U(2)_{L}$ doublets and right-handed singlets] are entirely induced by the signs in front of these bilinear brane terms. This new relation shows how the particular chiral properties of the SM could be explained by an underlying theory, through the bilinear brane term signs. We complete the analysis by a discussion, in this context, on the appropriate treatment of the cutoff in energy due to the framework of higher-dimensional models in a nonrenormalizable theory.

The paper is organized as follows. First, we describe the minimal model in Sec. II, before presenting the free case and the 4D treatment of the coupled fermions in Sec. III. The 5D approaches are exposed as well, with (Sec. IV) and without (Sec. V) regularization. Finally, an overview is provided in Sec. VI, together with a description of the phenomenological impacts. We summarize and conclude in the last section.

\section{MINIMAL CONSISTENT MODEL}

\section{A. Space-time structure}

We consider a 5D toy model with a space-time $\mathcal{E}^{5}=\mathcal{M}^{4} \times \mathcal{C}^{1}$ :

(i) $\mathcal{M}^{4}$ is the usual $4 \mathrm{D}$ Minkowski space-time. An event in $\mathcal{M}^{4}$ is characterized by its 4 -vector coordinates $x^{\mu}$ where $\mu=0,1,2,3$ is the Lorentz index. The metric and conventions used are given in Appendix A.

(ii) $\mathcal{C}^{1}$ is a finite one-dimensional flat compactified extra space. For our purpose, we consider the following simple case: the interval $\mathcal{C}^{1} \equiv[0, L]$, with a length $L \in \mathbb{R}_{+}^{\star}$, parametrized by the continuous extra coordinate $y$ and bounded by two flat 3-branes at $y=0$ and $y=L$.

(iii) A point of the whole 5D space-time $\mathcal{E}^{5}$ is labeled by its coordinates $z^{M}$ with an index $M \in \llbracket 0,4 \rrbracket \cdot z^{M}$ can be split into $\left(x^{\mu}, y\right)$.

\section{B. Bulk fermions}

We consider the minimal spin- $1 / 2$ fermion field content, allowing us to write down the 4D effective renormalizable SM Yukawa-like coupling between zero-mode fermions (of different chiralities) and a scalar field (see Sec. II E): a pair of fermions $Q$ and $D$. Both are propagating along the extra dimension, as we have in mind a model extension to a realistic scenario with bulk matter (cf. Sec. II F) where $Q$ and $D$ will be, respectively, the $S U(2)_{L}$ doublet downcomponent and singlet quark fields. The 5D fields $Q\left(x^{\mu}, y\right)$ and $D\left(x^{\mu}, y\right)$ thus have the kinetic terms in the covariant 5D action 


$$
\begin{aligned}
S_{\Psi}= & \int d^{4} x d y \frac{i}{2}\left(\bar{Q} \Gamma^{M} \partial_{M} Q-\partial_{M} \bar{Q} \Gamma^{M} Q\right. \\
& +\{Q \leftrightarrow D\}),
\end{aligned}
$$

where the last term indicates a field replacement and $\Gamma^{M}$ denotes the 5D Dirac matrices (see Appendix A). In our notations, the 5D Dirac spinor, being the irreducible representation of the Lorentz group, reads as

$$
Q=\left(\begin{array}{c}
Q_{L} \\
Q_{R}
\end{array}\right) \quad \text { and } \quad D=\left(\begin{array}{c}
D_{L} \\
D_{R}
\end{array}\right)
$$

in terms of the two two-component Weyl spinors, for the fields $Q$ and $D$, respectively. $L / R$ stands for the left/righthanded chirality.

Let us rewrite the bulk action of Eq. (2.1) in a convenient form. Using the definition $\overleftrightarrow{\partial_{M}} \hat{=} \overrightarrow{\partial_{M}}-\overleftrightarrow{\partial_{M}}$ and applying integrations by part along the usual 4-coordinates, it can be recast into $S_{\Psi}=\int d^{4} x d y \mathcal{L}_{\Psi}$ with

$$
\begin{aligned}
\mathcal{L}_{\Psi}= & \sum_{F=Q, D}\left\{i F_{R}^{\dagger} \sigma^{\mu} \partial_{\mu} F_{R}+i F_{L}^{\dagger} \bar{\sigma}^{\mu} \partial_{\mu} F_{L}\right. \\
& \left.+\frac{1}{2}\left(F_{R}^{\dagger} \stackrel{\leftrightarrow}{\partial_{4}} F_{L}-F_{L}^{\dagger} \stackrel{\leftrightarrow}{\partial_{4}} F_{R}\right)\right\}
\end{aligned}
$$

omitting the global 4-divergence, which must vanish in the action integration due to vanishing fields at (infinite) boundaries. Indeed, when minimizing the action, we see that the varied terms must vanish separately at (infinite) boundaries, since the generic nonvanishing field variations at boundaries are independent from each other and from bulk ones. This is realized by the standard configuration of vanishing fields themselves at boundaries, which is compatible with the wave function normalization conditions.

\section{Bilinear brane terms}

Interestingly, in the absence of the vanishing fermion current condition at a boundary of the considered interval $[0, L]$, the presence at this 3-brane of some bilinear terms, for bulk fermions being either free or coupled to a scalar field on this brane, turns out to be necessary. Indeed, these bilinear terms insure the existence of physical solutions (see Sec. III for the 4D approach and Sec. V for the 5D one) deduced from the least action principle. The theoretical reason for the presence of the bilinear brane terms (BBTs) at the boundaries of the interval is the correct geometrical configuration definition for models in which fermions cannot propagate beyond the two boundaries, as will also be described in Secs. III and V. These sections will also point out the 4D/5D matching of the mass spectrum exact result, which constitutes in particular a confirmation for the necessary presence and exact form (including coefficients) of the BBTs.
Necessary BBTs read as ${ }^{4}$

$$
\begin{aligned}
S_{\mathrm{B}} & =\int_{0}^{L} d^{4} x d y\left[\{\delta(y-L)-\delta(y)\} \mathcal{L}_{B}\right], \quad \text { with } \\
\mathcal{L}_{B} & =\frac{1}{2}(\bar{Q} Q-\bar{D} D),
\end{aligned}
$$

where $\delta(y-L)$ denotes the Dirac peak at $y=L$. Indeed, those BBTs will lead to the set of boundary conditions in Eq. (3.21) for the wave functions $q^{n}(y)$ and $d^{n}(y)$ of the 5D fields $Q$ and $D$, which then possess a nonvanishing normalizable zero mode $\left(m_{[n=0]}=0\right)$ for only one chirality [ $L$ or $R$ as $\sin \left(m_{[n=0]} y\right)=0$ ]; hence, at low energies (below the first KK mass eigenvalue $m_{1}$ ), only one chirality of a given 4D field arises in the KK decomposition (3.5) so that one recovers the chiral nature of the SM. Furthermore, within an extended realistic model (as described in Sec. II F) in which the $Q^{(D)}$ field would be the down-component of an SM $S U(2)_{L}$ gauge doublet, the unique chiralities of the zero-mode $4 \mathrm{D}$ fields $Q_{L}^{(D) 0}\left(x_{\mu}\right)$ and $D_{R}^{0}\left(x_{\mu}\right)$ predicted by Eq. (3.21) via Eq. (3.5) would correspond well to the SM chirality configuration. ${ }^{5}$ Notice that Eq. (3.5) (involving KK modes rather than mass eigenstates) and Eq. (3.21) are valid within the relevant $4 \mathrm{D}$ treatment of the localized Yukawa interaction where it is explicit that the SM particles (whose mass mainly originates from the EW symmetry breaking) are indeed mainly composed by the zero modes (small mixings with the massive KK states), as imposed by the small experimental deviations generally observed with respect to the theoretical SM predictions.

Therefore, it is remarkable that the BBTs allow us to make a step toward the UV explanation of the well-known SM chiral properties (chiral nature and chirality configuration) by directly linking these chiral aspects to explicit signs in front of Lagrangian terms (BBT signs), as described right above. Then, the last step would be to build a UV completion of the model to generate these BBT signs. In other words, the entire control of the chiral structure by the BBT signs is a new feature that shows how an underlying theory could produce the SM chiral structure.

For completeness, we mention that the two other BBT sign configurations,

\footnotetext{
${ }^{4}$ Similar terms, leading in particular to $\mathcal{L}_{B}=\frac{1}{2}\left(\bar{Q}^{D} Q^{D}-\bar{D} D\right)$, would hold in a model version extended to the EW symmetry of the SM, with the $Q$ field promoted to an $S U(2)_{L}$ doublet. In contrast, terms of the kind $\bar{Q}^{U} D$ (or $\bar{Q} D$ ), $\bar{Q}^{U} Q^{D}$, or $\bar{U} D$ would obviously not belong to a gauge-invariant form.

${ }^{5}$ Taking the opposite sign for each of the four terms in Eq. (2.4) would lead to exchanged boundary conditions between $q^{n}(y)$ and $d^{n}(y)$ relatively to Eq. (3.21) and in turn to another chirality configuration.
} 
$S_{\mathrm{B}}^{\prime}=\int_{0}^{L} d^{4} x d y\left[\{\delta(y-L)+\delta(y)\} \mathcal{L}_{B}^{\prime}\right], \quad$ with

$\mathcal{L}_{B}^{\prime}=\sigma \frac{1}{2} \bar{F} F$,

and $\sigma= \pm 1$, for $5 \mathrm{D}$ fields of the form (3.5) lead to the two sets (3.22) of boundary conditions and in turn to a vectorlike field content, as for the so-called custodian fermions in custodially protected warped models [39]. Indeed, Eq. (3.22) leads to the absence of zero modes $\left(m_{[n=0]} \neq 0\right)$ and hence any KK state has both left and right chiralities. Notice here that Eqs. (3.5) and (3.22) are valid for the free case. Once again, the control of the vectorial structure by the BBT signs is a novel characteristic that shows how a UV completion could produce a vectorlike field content.

What is the direct effect of the BBTs (2.4) on the final fermion mass eigenvalues? In the $4 \mathrm{D}$ approach and in the case without Yukawa interaction (see Sec. III), these BBTs have no effect on the 4D fermion mass matrix [Eq. (3.35)]; after injecting the profile solutions, those BBTs vanish due to the induced boundary conditions of Eq. (3.21), which impose that one of the two wave functions $(L \text { or } R)^{6}$ entering the BBT 5D fields [cf. Eq. (3.5)] is equal to zero, at $y=0 \quad\left[\sin \left(m_{n} 0\right)=0\right]$ and $y=L \quad\left[\sin \left(m_{n} L\right)=0\right]$, systematically for each one of the two Lagrangian BBTs (2.4). In contrast, in the 5D approach, the BBTs (2.4) play a numerical and direct role in the fermion mass spectrum, through the boundary conditions coming from the action variations (see Sec. V).

Formerly, this kind of bilinear fermion brane terms (2.4)-(2.5) was first introduced by hand to derive the more specific AdS/CFT correspondence in the calculation of correlation functions for spinors [40,41] - the exact AdS/CFT duality being possibly realized in the UV completion of warped models (from which the present simplified scenario is inspired). Then, within this AdS/CFT paradigm, similar boundary terms have been added at the UV-brane only $(y=0)$ to guarantee the minimization of the action in the holographic version of the warped model with bulk fermions [42]. The least action principle was also invoked in Ref. [43] to justify such bilinear fermion brane terms in the AdS/CFT context and through the path integral formalism. Equivalently, still in the AdS/CFT framework, these terms have been motivated in the Lagrangian density from an action form involving explicitly the Hamiltonian (to obtain a consistent Hamiltonian formulation when performing the Legendre transformation) [44]. Other boundary-localized terms were also introduced in a field theory defined on a manifold with boundaries within the context of gravity: the GibbonsHawking boundary terms [45-48]. Those terms are needed

\footnotetext{
${ }^{6}$ For instance, $\bar{D} D=D_{L}^{\dagger} D_{R}+D_{R}^{\dagger} D_{L}$.
}

to cancel the variation of the Ricci tensor at the boundaries of the manifold.

The finite geometry setup is defined via either the BBT inclusion or the vanishing fermion current condition, depending on the considered UV completion of the model. From the point of view of the effective field theory, it means that it can happen that the underlying theory does not forbid (through a short-distance mechanism or a residual symmetry) any possible nonrenormalizable Lorentz-invariant operator involving the 5D fields $Q$ and $D$ (including covariant derivatives) up to dimension 5-this dimension choice being motivated in Sec. II E-in the low-energy effective model described in this section. Then, the present fermionic operators would be those included in the considered actions (2.3) (dimension-5 operators) and (2.4) (dimension-4 operators): the BBT part.

Notice that bulk mass terms, usually modifying the bulk fermion profiles, bring useless complications, so we will not consider them in our present calculations, as the paper conclusions on fermion couplings to a brane field can be easily extended [49].

\section{Brane-localized scalar field}

The subtle aspects arise when the fermions couple to a single 4D scalar field, $H$, confined on a boundary taken here to be at $y=L$ (as inspired from warped scenarios addressing the gauge hierarchy problem). The action of this scalar field has the generic form

$$
\begin{aligned}
& S_{H}=\int d^{4} x d y \delta(y-L) \mathcal{L}_{\mathrm{H}}, \quad \text { with } \\
& \mathcal{L}_{\mathrm{H}}=\left(\partial_{\mu} H\right)^{\star}\left(\partial^{\mu} H\right)-V(H),
\end{aligned}
$$

where the potential $V$ possesses a minimum which generates a nonvanishing vacuum expectation value (VEV) for the field developed as

$$
H=\frac{v+h\left(x^{\mu}\right)}{\sqrt{2}}
$$

in analogy with the SM Higgs boson.

Note that one could think of replacing (up to a constant) the $\delta(y-L)$ peak in Eq. (2.6) by a Heaviside step ${ }^{7}$ function $\Theta(L-y)$ that could play a similar role of localizing the scalar field Lagrangian at the boundary $y=L$. Nevertheless, the integration in Eq. (2.6) over the interval $[0, L]$ would then be strictly equivalent to the integration over $[0, L]$ and in turn equal to zero given the vanishing Heaviside function value there. Such a situation would in fact correspond to the total absence of the $H$ scalar field, which conflicts with the considered field content hypothesis.

\footnotetext{
${ }^{7} \Theta(r)=0$ for $r>0$, and $\Theta(r) \hat{=} 1$ for $r=0$.
} 


\section{E. Yukawa-like interactions}

We focus on the following basic interaction in order to study the subtleties induced by the brane-scalar field coupling to bulk fermions:

$$
\begin{aligned}
& S_{\mathrm{Y}}=-\int d^{4} x d y \delta(y-L) \mathcal{L}_{\mathrm{Y}}, \quad \text { with } \\
& \mathcal{L}_{\mathrm{Y}}=Y_{5} Q_{L}^{\dagger} H D_{R}+Y_{5}^{\prime} Q_{R}^{\dagger} H D_{L}+\text { H.c. }
\end{aligned}
$$

Considering operators, involving $H, Q$, and $D$, up to dimension 5 allows us to include this Yukawa coupling of interest. ${ }^{8}$ The coupling constants $Y_{5}$ and $Y_{5}^{\prime}$ of Yukawa type, entering these two distinct terms, are independent (i.e., parameters with possibly different values) as a welldefined 4D chirality holds for the fermion fields on the 3-brane strictly at $y=L$ (see, for instance, Ref. [28]).

To avoid the introduction of a new energy scale, one could define the 5D Yukawa coupling constants by giving their explicit dependence in $L: Y_{5}=Y_{4} L$ and $Y_{5}^{\prime}=Y_{4}^{\prime} L$, where $Y_{4}, Y_{4}^{\prime}$ are dimensionless coupling constants of $\mathcal{O}(1)$. Then, $Y_{4}$ can be identified with the SM Yukawa coupling constant, as shown when applying the decoupling limit (infinitely heavy KK masses and any new physics energy scale).

From now on, we restrict our considerations to the VEV of $H$ as the aim is to calculate the $\mathrm{KK}$ fermion mass spectrum which is unaffected by the interactions of the $h\left(x^{\mu}\right)$ fluctuation field with fermions. Hence, we concentrate on the action issued from Eq. (2.7),

$$
\begin{aligned}
& S_{X}=-\int d^{4} x d y \delta(y-L) \mathcal{L}_{\mathrm{X}}, \quad \text { with } \\
& \mathcal{L}_{\mathrm{X}}=X Q_{L}^{\dagger} D_{R}+X^{\prime} Q_{R}^{\dagger} D_{L}+\text { H.c. }
\end{aligned}
$$

with the compact notations $X=\frac{v Y_{5}}{\sqrt{2}}$ and $X^{\prime}=\frac{v Y_{5}^{\prime}}{\sqrt{2}}$.

\section{F. Model extension}

The toy model considered is thus characterized by the Lagrangian

$$
S_{5 \mathrm{D}}=S_{\Psi}+S_{B}+S_{H}+S_{X} .
$$

Nevertheless, the conclusions of the present paper can be directly generalized to realistic warped models with bulk SM matter solving the fermion mass and gauge hierarchies. Indeed, working with a warp extra dimension instead of a

\footnotetext{
${ }^{8}$ Notice that, for instance, a dimension-6 operator of type $\frac{1}{M^{2}} \delta(y-L) Q_{L / R}^{\dagger} H^{2} D_{R / L}, M$ being a mass scale, would be treated in a similar way as the couplings in Eq. (2.7) [and can contribute to the Yukawa couplings (2.7) through the scalar field VEV].

${ }^{9}$ Note that in the decoupling limit where in particular $L \rightarrow 0$, generally $Y_{5} \rightarrow 0$ due to the dimension of the 5D Yukawa coupling constants.
}

flat one would not affect the conceptual subtleties about coupling bulk fermions to a brane-scalar field [49]. The boundaries at $y=0$ and $y=L$ could then become, respectively, the Planck and $\mathrm{TeV}$ branes. Similarly, the scalar potential, $V(H)$, can be extended to any potential (like the SM Higgs potential breaking the EW symmetry) as long as it still generates a VEV for the scalar field as here. In this context, the $H$ singlet can be promoted to the Higgs doublet under the SM $S U(2)_{L}$ gauge group, simply by inserting doublets in the kinetic term of Eq. (2.6). The whole structure of the coupling of Eq. (2.8) between bulk fermions and the localized VEV would as well remain identical in case of fermions promoted to SM $S U(2)_{L}$ doublets: after group contraction of the doublet $\left(Q^{U}, Q^{D}\right)^{t}$ with down-/up-quark singlets $D, U$, one would obtain two replicas of the structure (2.8) with the forms $Q_{C}^{D^{\dagger}} D_{C^{\prime}}$ and $Q_{C}^{U^{\dagger}} U_{C^{\prime}}$, where $C^{(\prime)} \equiv L, R$ denotes the chirality. Hence, the procedure described in this paper should just be applied to both terms separately. ${ }^{10}$ The same comment holds for the SM color triplet contraction and the field content extension to the three flavors of quarks and leptons of the SM. Notice that the flavor mixings would be combined with the mixings among fermion modes of the KK towers, without any impact on the present considerations about branelocalized couplings.

\section{4D PERTURBATIVE APPROACH}

\section{A. 5D aspects for (formally) free fermions}

In this part, we calculate the fermionic mass spectrum in the basic case in which $Y_{5}=Y_{5}^{\prime}=0$ in Eq. (2.7) (studied in various references $[27,35,42,50-57])$, pointing out the correct treatment. Let us also remark that in this case there is no $4 \mathrm{D} / 5 \mathrm{D}$ matching condition to look at (pure 5D calculation of the masses). The main interest of this section is to develop the rigorous procedure for applying the boundary conditions.

\section{Absence of Yukawa couplings}

Natural boundary conditions.-To extract from the relevant Lagrangian (2.3) the equations of motion (EOM) and the boundary conditions (BCs) for the bulk fermions, we apply the least action principle-or Hamilton's variational principle-for each of them $\left(F=Q, D^{11}\right)$. Assuming, at a first level, the boundary fields $\left.F\left(x^{\mu}, y=\{0, L\}\right) \hat{=} F\right|_{0, L}$ to be initially unknown (unfixed), they should be deduced from the action minimization with respect to them, considering thus nonvanishing generic ${ }^{12}$ variations

\footnotetext{
${ }^{10}$ The fermion actions in Eqs. (2.3) and (2.4) would be trivially generalized as well to a scenario with a gauge symmetry.

${ }^{11} \mathrm{EOM}$ and $\mathrm{BCs}$ for the fields and their conjugates are trivially related through Hermitian conjugation.

${ }^{12} \mathrm{~A}$ field variation reads as $\delta F\left(z^{M}\right)=\epsilon \eta\left(z^{M}\right)$ with a generic function $\eta\left(z^{M}\right)$ and an infinitesimal parameter $\epsilon \rightarrow 0$.
} 
$\left.\delta F\right|_{0, L} \neq 0 .{ }^{13}$ In other words, $\left.F\right|_{0, L}$ should be then obtained from the so-called natural boundary conditions (NBCs). The stationary action condition can be split, without loss of generality (the functional variations are generic so that $\delta \bar{Q}$ and $\delta \bar{D}$ are independent), into action variations with respect to each field $\bar{Q}$ and $\bar{D}$,

$$
\begin{aligned}
0 & =\delta_{\bar{F}} S_{\Psi} \\
& =\int d^{4} x \int_{0}^{L} d y\left[\delta \bar{F} i \Gamma^{M} \partial_{M} F+\frac{1}{2} \partial_{4}\left(\delta \bar{F} \gamma^{5} F\right)\right],
\end{aligned}
$$

as written after we have expressed Eq. (2.3) in terms of the four-component spinors, based on the Dirac matrices of Appendix A, and integrated by part its last two terms. Note that $\delta\left(\partial_{M} \bar{F}^{\alpha}\right) \partial \mathcal{L}_{\Psi} / \partial\left(\partial_{M} \bar{F}^{\alpha}\right)=0$ ( $\alpha$ being the implicitly summed spinor index) was used to obtain Eq. (3.1). Besides, the variations $\left.\delta \bar{F}\right|_{0, L} \neq 0$ appear in the second term of this equation (pure boundary terms after integration over $y$ of the global $\partial_{4}$ derivative) generated by the action minimization with respect to $\bar{F}$ at the boundaries.

Following the theory of variational calculus $[58,59]$, the distinct terms of Eq. (3.1) vanish separately (respectively, the volume and the surface terms) to insure that $\delta_{\bar{F}} S_{\Psi}=0$ still for generic and in turn independent field variations,

$$
\delta_{\bar{F}} S_{\Psi}=0 \Rightarrow \begin{cases}0=\delta \bar{F} i \Gamma^{M} \partial_{M} F, & \forall x^{\mu}, \forall y \in[0, L] \\ 0=\left.\delta \bar{F} \gamma^{5} F\right|_{0}=\left.\delta \bar{F} \gamma^{5} F\right|_{L}, & \forall x^{\mu},\end{cases}
$$

where the first (second) line constitutes the bulk EOM (NBCs). ${ }^{14}$ Notice that the NBCs originate from the last term of Eq. (3.1) obtained after an integration by parts of the initial action. Using once more the fact that for the searched bulk fields the $\delta \bar{F}^{\alpha}\left(z^{M}\right)$ for any $\delta \bar{F}^{\alpha}[\alpha=0,1, \ldots, 4]$ are independent from each other and nonvanishing, it is useful for the following to recast Eq. (3.2) into these twocomponent spinor relations (still using Appendix A),

$$
i \Gamma^{M} \partial_{M} F=0 \Leftrightarrow\left[\begin{array}{l}
i \sigma^{\mu} \partial_{\mu} F_{R}=-\partial_{4} F_{L} \\
i \bar{\sigma}^{\mu} \partial_{\mu} F_{L}=\partial_{4} F_{R}
\end{array}\right]
$$

and

\footnotetext{
${ }^{13}$ Then, in the final step, once, for instance, the field $\left.F\right|_{L}$ is found and fixed by the solution (not initially fixed as an hypothesis in this considered case), its resulting determined form does not imply $\left.\delta F\right|_{L}=0$, which would be incompatible with the starting nonvanishing field variation; there is sometimes confusion in articles about these chronological aspects of the variational calculus.

${ }^{14}$ We find the Hermitian conjugate EOM and NBCs by integrating by parts the bulk piece of the relation $\delta_{F} S_{\Psi}=0$ (nonvanishing boundary terms from integration over the extra dimension then contribute) in order to get rid of the field factors $\partial_{M} \delta F$.
}

$\left.\gamma^{5} F\right|_{0}=\left.\gamma^{5} F\right|_{L}=\left.0 \Leftrightarrow F_{L}\right|_{0}=\left.F_{R}\right|_{0}=\left.F_{L}\right|_{L}=\left.F_{R}\right|_{L}=0$.

Let us now deduce, from those equations involving the 5D fields, the relations on their profiles along the extra dimension.

Naive approach.-To develop a 4D effective picture, let us replace the 5D fields, in the relations obtained just above, by their standard solution in the form of a $\mathrm{KK}$ decomposition,

$$
F_{L / R}\left(x_{\mu}, y\right)=\frac{1}{\sqrt{L}} \sum_{n=0}^{+\infty} f_{L / R}^{n}(y) F_{L / R}^{n}\left(x_{\mu}\right),
$$

where $f_{L / R}^{n}=q_{L / R}^{n}$ or $d_{L / R}^{n}$ are the dimensionless wave functions along the extra dimension associated, respectively, to the $4 \mathrm{D}$ fields $F_{L / R}^{n}=Q_{L / R}^{n}$ or $D_{L / R}^{n}$ of the $\mathrm{KK}$ excitations. The integer $n$ is defined throughout the whole paper as being the level index of the heavy fermion mode (here the KK state ${ }^{15}$ ) tower and is chosen to be positive; the meaningful feature about the general KK decomposition (3.5) is rather the infinite summation (possibly also from $-\infty$ to $+\infty$ ) dictated by field expressions as Fourier series on a finite interval.

Inserting Eq. (3.5) into the 5D field relations (3.3) and using the following two-component Weyl equations for the 4D fermions (issued from the four-component Dirac equation), ${ }^{16}$

$$
\left\{\begin{array}{l}
i \bar{\sigma}^{\mu} \partial_{\mu} F_{L}^{n}\left(x_{\mu}\right)=m_{n} F_{R}^{n}\left(x_{\mu}\right) \\
i \sigma^{\mu} \partial_{\mu} F_{R}^{n}\left(x_{\mu}\right)=m_{n} F_{L}^{n}\left(x_{\mu}\right)
\end{array},\right.
$$

where $m_{n}$ are the KK masses ${ }^{17}$ for the fermions, ${ }^{18}$ one can directly extract these differential free equations for the profiles:

$$
\forall n \geq 0, \quad\left\{\begin{array}{l}
\partial_{4} q_{R}^{n}(y)=m_{n} q_{L}^{n}(y) \\
\partial_{4} q_{L}^{n}(y)=-m_{n} q_{R}^{n}(y) \\
\partial_{4} d_{R}^{n}(y)=m_{n} d_{L}^{n}(y) \\
\partial_{4} d_{L}^{n}(y)=-m_{n} d_{R}^{n}(y)
\end{array}\right.
$$

These four equations have been obtained by writing the equality, per KK level, between each term of the KK state sums on the left-hand side and right-hand side of Eq. (3.3) (and by simplifying thanks to identical 4D fields on each side), instead of considering compensations between

\footnotetext{
${ }^{15}$ Not yet the mass eigenstate in case of Yukawa interactions.

${ }^{16}$ Within the natural unit system.

${ }^{17}$ Also mass eigenvalues in the absence of Yukawa interactions.

${ }^{18}$ The same masses $m_{n}$ enter the Weyl equations for the $Q_{L / R}^{n}\left(x_{\mu}\right)$ and $D_{L / R}^{n}\left(x_{\mu}\right)$ fields, which are described by separate and identical terms in the considered Lagrangian.
} 
different terms in Eq. (3.3), which would mean having physical 4D fields $F_{L / R}^{n}\left(x_{\mu}\right)$ [solutions of Eq. (3.6)] expressed as linear combinations of other mass eigenstates $F_{L / R}^{n^{\prime}}\left(x_{\mu}\right)$; such reexpressions would induce, in the Lagrangian, $\mathrm{KK}$ mass mixing terms for the mass eigenstates, which is not consistent.

Deriving and combining the first-order differential equations (3.7), one can decouple them into the second-order differential equations

$$
\forall n \geq 0, \quad \partial_{4}^{2} f_{L / R}^{n}(y)=-m_{n}^{2} f_{L / R}^{n}(y)
$$

being the equations of independent harmonic oscillators, whose solutions possess the general form

$\forall n \geq 0, f_{L / R}^{n}(y)=A_{L / R}^{n} \cos \left(m_{n} y\right)+B_{L / R}^{n} \sin \left(m_{n} y\right)$,

where $A_{L / R}^{n}$ and $B_{L / R}^{n}$ are constant parameters.

Now, inserting Eq. (3.5) into the 5D field conditions of Eq. (3.4), we obtain the vanishing conditions ${ }^{19}$ for any profile,

$$
\forall n \geq 0, \quad f_{L / R}^{n}(0)=f_{L / R}^{n}(L)=0,
$$

avoiding inconsistent relations among mass eigenstate 4D fields, as explained below Eq. (3.7).

Those conditions, combined with Eqs. (3.7) and (3.9), give rise to the vanishing profiles $f_{L / R}^{n}(y)=0(\forall n \geq 0),{ }^{20}$ which are not compatible with the two orthonormalization conditions for $f_{L}^{n}(y)$ and $f_{R}^{n}(y)$,

$\frac{1}{L} \int_{0}^{L} d y f_{L / R}^{n \star}(y) f_{L / R}^{m}(y)=\delta_{n m}, \quad \forall n \geq 0, \quad \forall m \geq 0$,

coming out from the imposition of canonical and diagonal normalized kinetic terms for the 4D fields after inserting the KK decomposition (3.5) into the 5D field kinetic terms (2.1).

The theoretical inconsistency obtained here for the considered free model reveals a problem in the treatment of a simple boundary without localized couplings to bulk matter (which is the case of both boundaries here). The correct treatments, based on either fermion current conditions at the boundaries or boundary-localized terms (the BBTs), are exposed respectively in the two following subsections.

\section{Introducing the fermion current condition}

Current from action variations. - In fact, the free version $\left[Y_{5}=Y_{5}^{\prime}=0\right]$ of the model defined in Sec. II (and finite

\footnotetext{
${ }^{19}$ Throughout this paper, the notation " $\forall n \geq 0$ " applies on the natural integer $\mathrm{n}$ defined in Eq. (3.5): $n=0,1,2,3, \ldots$.

${ }^{20} f_{L}^{n}(0)=0, \forall n$, and Eq. (3.9) leads to $A_{L}^{n}=0$ so that $f_{L}^{n}(y)=B_{L}^{n} \sin \left(m_{n} y\right)$. Then, Eq. (3.7) induces $f_{R}^{n}(y)=$ $A_{R}^{n} \cos \left(m_{n} y\right)$ and in turn $f_{R}^{n}(y)=0$ from $f_{R}^{n}(0)=0$. Hence, Eq. (3.7) imposes $B_{L}^{n}=0=f_{L}^{n}(y)$
}

extra dimension scenarios in general) does impose conditions on the bulk fermions at the extra dimension boundaries, which were not included in the above naive analysis. These conditions contribute to defining the geometrical field configuration of the considered model. They will constitute the so-called essential boundary conditions (EBCs), as imposed by the model definition, which are complementary to the NBC already defined in Eq. (3.2). Indeed, the NBCs come from an integration by parts of the initial action with respect to the fifth dimension over the interval $[0, L]$ and thus take into account the spacetime structure itself.

Regarding the geometrical field configuration within the present free model, each fermion field is defined only along the interval $[0, L]$. This model building hypothesis, that fermions neither propagate toward nor come from the outside of a finite range, translates into the condition of vanishing probability current at both boundaries for each independent fermion species separately (without possible compensations).

Formally speaking, after having varied the Lagrangian (2.1) [see Eq. (3.1)] and in turn derived the bulk EOM (3.3) [from the first relation of Eq. (3.2)] as well as the NBCs [second relation of Eq. (3.2)], the application of the Noether theorem demonstrated in Appendix B (by using the $\mathrm{EOM})^{21}$ gives rise to the two probability currents (B4) defined independently for the two bulk fermions ${ }^{22}$ represented by the $5 \mathrm{D}$ fields $F=Q, D$,

$$
j_{Q}^{M}=-\alpha \bar{Q} \Gamma^{M} Q, \quad j_{D}^{M}=-\alpha^{\prime} \bar{D} \Gamma^{M} D,
$$

associated to the two global $U(1)_{F}$ symmetries of the free action (2.1) corresponding, respectively, to the distinct transformations,

$$
Q \mapsto \mathrm{e}^{i \alpha} Q, \quad D \mapsto \mathrm{e}^{i \alpha^{\prime}} D .
$$

$\alpha, \alpha^{\prime}(\in \mathbb{R})$ are continuous parameters entering, for instance, the infinitesimal field variations: $:^{23}$

$$
\underline{\delta} Q=i \alpha Q, \quad \underline{\delta} \bar{Q}=-i \alpha \bar{Q} .
$$

Now, the four conditions of vanishing probability currents are thus

$$
\begin{aligned}
\left.j_{F}^{4}\right|_{0, L} & =-\left.\alpha^{(\prime)} \bar{F} \Gamma^{4} F\right|_{0, L}=\left.i \alpha^{(\prime)}\left(F_{R}^{\dagger} F_{L}-F_{L}^{\dagger} F_{R}\right)\right|_{0, L} \\
& =0, \quad \forall x^{\mu},
\end{aligned}
$$

where we have used Eq. (2.2). For a nontrivial transformation (3.13) $\left[\alpha^{(\prime)} \neq 0\right]$, the condition (3.15) on the 4D

\footnotetext{
${ }^{21}$ Valid trivially in the absence of BBTs as well.

${ }^{22}$ See Ref. [50] for scalar field currents.

${ }^{23} \mathrm{We}$ use different notations for the infinitesimal field variations under specific transformations, $\underline{\delta} F$, and generic field variations in the variation calculus, $\delta F$.
} 
fields $F_{L / R}^{n}\left(x_{\mu}\right)$ [cf. Eq. (3.5)] entirely fixed by Eq. (3.6) leads to an equation on the 4D space-time coordinates and momenta, whereas the fields of the considered model must be defined in the whole $4 \mathrm{D}$ space-time and for any $4 \mathrm{D}$ momentum. The most general way out is to make of Eq. (3.15) a trivial equality by having

$$
\begin{array}{lll}
\left.F_{L}\right|_{0}=0 & \text { or } & \left.F_{R}\right|_{0}=0, \quad \text { and }, \\
\left.F_{L}\right|_{L}=0 & \text { or } & \left.F_{R}\right|_{L}=0
\end{array}
$$

corresponding to a vanishing coefficient in each term of the condition (3.15),

$$
\begin{array}{ll}
f_{L}^{n}(0)=0 & \text { or } \quad f_{R}^{n}(0)=0 \quad \text { and } \\
f_{L}^{n}(L)=0 & \text { or } \quad f_{R}^{n}(L)=0, \quad \forall n \geq 0,
\end{array}
$$

which avoids inconsistent relations among mass eigenstate 4D fields, as discussed below Eq. (3.7).

These necessary conditions (3.16) of vanishing fields at boundaries are the EBCs and correspond to some fields initially fixed at boundaries. Having such known fields at boundaries imposes [58] having vanishing functional variations,

$$
\begin{array}{llll}
\left.\delta F_{L}\right|_{0}=0 & \text { or } & \left.\delta F_{R}\right|_{0}=0, & \text { and }, \\
\left.\delta F_{L}\right|_{L}=0 & \text { or } & \left.\delta F_{R}\right|_{L}=0 .
\end{array}
$$

There is an overall consistency since no action minimization with respect to a field $\left.F_{L / R}\right|_{0, L}$ (relying on $\left.\delta F_{L / R}\right|_{0, L} \neq 0$ ) is needed for such a known fermion field at a boundary, in contrast with the first treatment (above) where the boundary fields $\left.F\right|_{0, L}$ were assumed to be initially unknown and then found out (NBCs) through the least action principle.

The new EBCs (3.16) must be combined with the obtained NBCs [second relation of Eq. (3.2)], which read as

$$
\left.\left(\delta F_{L}^{\dagger} F_{R}\right)\right|_{0}-\left.\left(\delta F_{R}^{\dagger} F_{L}\right)\right|_{0}=\left.\left(\delta F_{L}^{\dagger} F_{R}\right)\right|_{L}-\left.\left(\delta F_{R}^{\dagger} F_{L}\right)\right|_{L}=0 .
$$

In fact, each of the four sets of EBCs in Eqs. (3.16)-(3.18) imply the NBCs (3.19) so that it is sufficient to consider these EBCs.

In other words, when deriving the NBCs, before knowing the EBCs (as described above), one would sum generically in the action variations (3.1) the terms with all nonvanishing field variations at boundaries. Once the EBCs (3.16) would be determined and selected [fixing some fields at boundaries accordingly to Eq. (3.18)], one would keep only the known nonvanishing variations (i.e., omit to vary the action with respect to known fields) and in turn one would do the same in the NBC [so that some terms of Eq. (3.19) would be omitted].
Now, the general solutions (3.9) of the decoupled equations derived from the EOM (3.3), once reinjected into the initial equations (3.7) on the profiles, become

$$
\begin{aligned}
& f_{L}^{n}(y)=B_{R}^{n} \cos \left(m_{n} y\right)-B_{L}^{n} \sin \left(m_{n} y\right), \\
& f_{R}^{n}(y)=B_{L}^{n} \cos \left(m_{n} y\right)+B_{R}^{n} \sin \left(m_{n} y\right) .
\end{aligned}
$$

These solutions are taken continuous at the boundaries in order to possibly have well-defined derivatives appearing in the consistent action (2.1), as also described in details in Sec. IV B 1. Applying the four sets of EBCs from Eqs. (3.16) and (3.17) to the solution forms (3.20), it appears that certain constant parameters must be equal to zero, and thus we obtain the following four possible sets of profiles and KK mass spectrum equation $(\forall n \geq 0)$,

$$
\begin{aligned}
\text { 1) }(--): d_{L}^{n}(y) & =-B_{L}^{n} \sin \left(m_{n} y\right), \\
(++): d_{R}^{n}(y) & =B_{L}^{n} \cos \left(m_{n} y\right) ; \sin \left(m_{n} L\right)=0, \\
\text { 2) }(++): q_{L}^{n}(y) & =B_{R}^{n} \cos \left(m_{n} y\right), \\
(--): q_{R}^{n}(y) & =B_{R}^{n} \sin \left(m_{n} y\right) ; \sin \left(m_{n} L\right)=0,
\end{aligned}
$$

and

$$
\text { 3) } \begin{aligned}
(-+) & : f_{L}^{n}(y)=-B_{L}^{n} \sin \left(m_{n} y\right), \\
(+-) & : f_{R}^{n}(y)=B_{L}^{n} \cos \left(m_{n} y\right) ; \cos \left(m_{n} L\right)=0, \\
\text { 4) }(+-): f_{L}^{n}(y) & =B_{R}^{n} \cos \left(m_{n} y\right), \\
(-+) & : f_{R}^{n}(y)=B_{R}^{n} \sin \left(m_{n} y\right) ; \cos \left(m_{n} L\right)=0 .
\end{aligned}
$$

Here, we have used the standard BC notations; i.e., - or +, for example, at $y=0$ stands, respectively, for the Dirichlet or Neumann wave function BC: $f_{L / R}^{n}(0)=0$ or $\partial_{y} f_{L / R}^{n}(0)=0$. For instance, the symbolic notation $(-+)$ denotes the Dirichlet (Neumann) BC at $y=0$ $(y=L)$. The solutions (3.21) assigned to the (singlet/ doublet component) quark fields give rise to the chiral nature of the SM and to its correct chirality configuration, as described in Sec. II C for Eq. (3.21). The other solutions (3.22) lead to KK towers without zero modes like custodian states [see also the discussion on Eq. (3.22) in Sec. II C].

Notice that the used BCs (3.17) must be injected into the Eqs. (3.7) issued from the EOM as those are valid for any point of the extra dimension including the boundaries [see the original Eq. (3.2)]. This leads to a new set of BCs that we call the complete BCs. These complete BCs are well satisfied by the final solutions (3.21) and (3.22).

The constants $B_{L}^{n}=\sqrt{2} e^{i \alpha_{L}^{n}}$ and $B_{R}^{n}=\sqrt{2} e^{i \alpha_{R}^{n}}$ $(\forall n>0),{ }^{24}$ where $\alpha_{L / R}^{n}$ are real angles, are fixed by the orthonormalization condition (3.11). The relation

\footnotetext{
${ }^{24}$ For solution 1 , we find $B_{L}^{0}=e^{i \alpha_{L}^{0}}$, while $B_{R}^{0}=e^{i \alpha_{R}^{0}}$ for solution 2 [cf. Eq. (3.21)].
} 
$\sin \left(m_{n} L\right)=0$ has the following chosen solutions for the KK mass spectrum:

$$
m_{n}=+\frac{n \pi}{L} \quad(\forall n \geq 0)
$$

The spectrum $m_{n}=-\frac{n \pi}{L}[\forall n \geq 0]$ is also possible. Similarly, the relation $\cos \left(m_{n} L\right)=0$ has the possible solutions $m_{n}= \pm\left(\frac{\pi}{2 L}+\frac{n \pi}{L}\right)[\forall n \geq 0]$.

Current from equations of motion.-Alternatively, as the starting point, one can apply the vanishing conditions (3.15) (EBCs) on the same probability currents (3.12) (up to the definition constant $-\alpha^{(/)}$) satisfying the conservation relations, $\partial_{M} j_{F}^{M}=0$, as derived directly (without applying the Noether theorem) from a rewriting ${ }^{25}$ of each free 5D Dirac equation (3.3) in the bulk:

$$
i \Gamma^{M} \partial_{M} Q=0, \quad i \Gamma^{M} \partial_{M} D=0 .
$$

To derive possible NBCs, one now has to consider the action. The free bulk fermion action can be rewritten, after an integration by parts in the last two terms of Eq. (2.3), as

$$
\begin{aligned}
S_{\Psi}= & \int d^{4} x d y \sum_{F=Q, D}\left\{i F_{R}^{\dagger} \sigma^{\mu} \partial_{\mu} F_{R}+i F_{L}^{\dagger} \bar{\sigma}^{\mu} \partial_{\mu} F_{L}\right. \\
& +F_{R}^{\dagger} \partial_{4} F_{L}-F_{L}^{\dagger} \partial_{4} F_{R} \\
& \left.+\frac{1}{2}[\delta(y-L)-\delta(y)]\left(F_{L}^{\dagger} F_{R}-F_{R}^{\dagger} F_{L}\right)\right\} .
\end{aligned}
$$

Injecting directly the EBCs (3.15) into the Lagrangian would cancel out the boundary terms of Eq. (3.25) and in turn spoil the necessary Hermiticity of the action [being explicit through Eqs. (2.1)-(2.3)]. This feature reflects the fact that the action and the current condition (3.15) are distinct ingredients defining the model. The proper method goes as follows: the current condition (3.15) constitutes the EBCs which will have to be combined with the action minimization relations.

So, let us apply the least action principle to the action (3.25). From the known EBCs (3.15), leading to the conditions (3.16), we deduce that the field $\left.F_{L}\right|_{0}$ or $\left.F_{R}\right|_{0}$ is fixed to zero, as is the field $\left.F_{L}\right|_{L}$ or $\left.F_{R}\right|_{L}$, so that their functional variation vanishes [as in Eq. (3.18)] and in turn the global variation of the action part in the second line of Eq. (3.25) cancels out. Another way to find out this cancellation is to combine the functional variation of the relation (3.15) with the variation of the terms in the second line of Eq. (3.25). Therefore, the action minimization only

\footnotetext{
${ }^{25}$ Subtracting the Dirac equation to its Hermitian conjugate form, with the relevant 5D field and $\gamma^{0}$ factors, and using the 5D Dirac matrix rules.
}

leads to the same bulk EOM as in Eq. (3.3). These bulk equations induce the profile equations (3.7) whose solutions have the forms given by Eq. (3.20), which are taken once more continuous at the boundaries. As above, the EBCs (3.15)-(3.17) applied to these solutions give rise to the final profiles (3.21) and (3.22) and in turn to the mass spectrum discussed via Eq. (3.23).

\section{Introducing the bilinear brane terms (NBCs)}

As announced at the end of Sec. III A 1, an alternative method $^{26}$ with respect to previous subsection for finding out the same consistent physical solutions, for the mass spectrum and the profiles, is to add the BBTs (2.4) to the kinetic terms (2.3) so that the initial free fermionic action becomes

$$
S_{5 \mathrm{D}}^{\mathrm{free}}=S_{\Psi}+S_{B}
$$

Let us apply the least action principle using this action as the starting point. The action based on Eq. (2.3), rewritten as the action (3.25) and added to the BBT piece (2.4), reads as, after combining the boundary terms,

$$
\begin{aligned}
S_{5 \mathrm{D}}^{\mathrm{free}}= & \int d^{4} x d y \sum_{F=Q, D}\left\{i F_{R}^{\dagger} \sigma^{\mu} \partial_{\mu} F_{R}+i F_{L}^{\dagger} \bar{\sigma}^{\mu} \partial_{\mu} F_{L}\right. \\
& \left.+F_{R}^{\dagger} \partial_{4} F_{L}-F_{L}^{\dagger} \partial_{4} F_{R}\right\} \\
& +\int d^{4} x d y[\delta(y-L)-\delta(y)]\left(Q_{L}^{\dagger} Q_{R}-D_{R}^{\dagger} D_{L}\right) .
\end{aligned}
$$

Without loss of generality, the stationary action condition can be split into these two conditions with respect to the two field variations, respectively,

$$
\begin{aligned}
0=\delta_{F_{L}^{\dagger}} S_{5 \mathrm{D}}^{\text {free }} & =\int d^{4} x \int_{0}^{L} d y\left[\delta F_{L}^{\dagger} i \bar{\sigma}^{\mu} \partial_{\mu} F_{L}-\delta F_{L}^{\dagger} \partial_{4} F_{R}\right] \\
& +\int d^{4} x C_{F}^{L}\left[\left.\left(\delta F_{L}^{\dagger} F_{R}\right)\right|_{L}-\left.\left(\delta F_{L}^{\dagger} F_{R}\right)\right|_{0}\right], \quad \\
0=\delta_{F_{R}^{\dagger}} S_{5 \mathrm{D}}^{\text {free }} & =\int d^{4} x \int_{0}^{L} d y\left[\delta F_{R}^{\dagger} i \sigma^{\mu} \partial_{\mu} F_{R}+\delta F_{R}^{\dagger} \partial_{4} F_{L}\right] \\
& +\int d^{4} x C_{F}^{R}\left[-\left.\left(\delta F_{R}^{\dagger} F_{L}\right)\right|_{L}+\left.\left(\delta F_{R}^{\dagger} F_{L}\right)\right|_{0}\right]
\end{aligned}
$$

where $C_{D}^{L}=0, C_{D}^{R}=1, C_{Q}^{L}=1, C_{Q}^{R}=0$, for each field $D$ and $Q$. For generic field variations $\delta F_{L / R}^{\dagger}$ and $\left.\delta F_{L / R}^{\dagger}\right|_{0, L}$, the sum of the first two terms, both in Eqs. (3.27) and (3.28), must vanish separately, leading to the same equations as the

\footnotetext{
${ }^{26}$ One could simultaneously impose the EBC (3.15) above and add the BBT to the action, but this method would contain some redundancy.
} 
EOM $(3.3)^{27}$ and in turn via Eq. (3.6) to the profile equations (3.7) with solutions (3.9). The general solutions (3.9), once injected into the initial equations (3.7), take the specific forms (3.20). We are thus left with the NBCs:

$$
\begin{aligned}
& \left.C_{F}^{L}\left(\delta F_{L}^{\dagger} F_{R}\right)\right|_{L}=0,\left.\quad C_{F}^{L}\left(\delta F_{L}^{\dagger} F_{R}\right)\right|_{0}=0, \\
& \left.C_{F}^{R}\left(\delta F_{R}^{\dagger} F_{L}\right)\right|_{L}=0 \quad \text { and }\left.\quad C_{F}^{R}\left(\delta F_{R}^{\dagger} F_{L}\right)\right|_{0}=0 .
\end{aligned}
$$

Then, using the appropriate constants $C_{Q}^{L, R}$ and $C_{D}^{L, R}$ above for each field and generic variations $\left.\delta F_{L, R}^{(\dagger)}\right|_{0, L} \neq 0$, it appears clearly that those BCs belong to the set of BCs (3.16) and (3.17) whose application on the solution forms (3.20) leads to the two respective sets of profiles and KK mass spectrum (3.21), as already derived. The structure of the profile solutions (3.21) corresponds to the chiral nature and configuration of the SM as already explained in Sec. II C.

For completeness, starting instead from the BBT (2.5) in the action for a given field $F$,

$$
S_{5 \mathrm{D}}^{\text {ffree }}=S_{\Psi}(F)+S_{B}^{\prime},
$$

the combination of the boundary terms leads to

$$
\begin{aligned}
S_{5 \mathrm{D}}^{\text {ffree }}= & \int d^{4} x d y\left\{i F_{R}^{\dagger} \sigma^{\mu} \partial_{\mu} F_{R}+i F_{L}^{\dagger} \bar{\sigma}^{\mu} \partial_{\mu} F_{L}\right. \\
& +F_{R}^{\dagger} \partial_{4} F_{L}-F_{L}^{\dagger} \partial_{4} F_{R} \\
& +\left[\delta(y-L) \frac{\sigma+1}{2}+\delta(y) \frac{\sigma-1}{2}\right] F_{L}^{\dagger} F_{R} \\
& \left.+\left[\delta(y-L) \frac{\sigma-1}{2}+\delta(y) \frac{\sigma+1}{2}\right] F_{R}^{\dagger} F_{L}\right\} .
\end{aligned}
$$

The stationary action condition can be split into the two following conditions:

$$
\begin{aligned}
0=\delta_{F_{L}^{\dagger}} S_{5 \mathrm{D}}^{\prime \text { free }} & =\int d^{4} x \int_{0}^{L} d y\left[\delta F_{L}^{\dagger} i \bar{\sigma}^{\mu} \partial_{\mu} F_{L}-\delta F_{L}^{\dagger} \partial_{4} F_{R}\right] \\
& +\int d^{4} x\left[\left.\frac{\sigma+1}{2}\left(\delta F_{L}^{\dagger} F_{R}\right)\right|_{L}+\left.\frac{\sigma-1}{2}\left(\delta F_{L}^{\dagger} F_{R}\right)\right|_{0}\right],
\end{aligned}
$$

$$
\begin{aligned}
0=\delta_{F_{R}^{\dagger}} S_{5 \mathrm{D}}^{\prime \text { free }} & =\int d^{4} x \int_{0}^{L} d y\left[\delta F_{R}^{\dagger} i \sigma^{\mu} \partial_{\mu} F_{R}+\delta F_{R}^{\dagger} \partial_{4} F_{L}\right] \\
& +\int d^{4} x\left[\left.\frac{\sigma-1}{2}\left(\delta F_{R}^{\dagger} F_{L}\right)\right|_{L}+\left.\frac{\sigma+1}{2}\left(\delta F_{R}^{\dagger} F_{L}\right)\right|_{0}\right] .
\end{aligned}
$$

\footnotetext{
${ }^{27}$ We obtain the Hermitian conjugate EOM and NBCs by integrating by parts the bulk piece of the relation $\delta_{F_{L, R}} S_{5 \mathrm{D}}^{\text {free }}=0$ (nonvanishing boundary terms appear due to the integration over the extra dimension) in order to get rid of the field factors $\partial_{M} \delta F_{L, R}$.
}

Once more, the sum of the first two terms in Eqs. (3.31) and (3.32), respectively, must vanish, leading to the same profile equations as the ones deduced from Eqs. (3.27) and (3.28) and hence to the identical bulk solution forms (3.20). Nevertheless, we are now left with the new NBCs:

$$
\begin{aligned}
\left.\frac{\sigma+1}{2}\left(\delta F_{L}^{\dagger} F_{R}\right)\right|_{L} & =\left.\frac{\sigma-1}{2}\left(\delta F_{L}^{\dagger} F_{R}\right)\right|_{0}=\left.\frac{\sigma-1}{2}\left(\delta F_{R}^{\dagger} F_{L}\right)\right|_{L} \\
& =\left.\frac{\sigma+1}{2}\left(\delta F_{R}^{\dagger} F_{L}\right)\right|_{0}=0
\end{aligned}
$$

Then, for generic variations $\left.\delta F_{L, R}^{(\dagger)}\right|_{0, L} \neq 0$, it is clear that those BCs belong to the set of BCs (3.16) and (3.17) whose application on the solution forms (3.20) leads, for $\sigma=+1$, to set 3 of profiles and KK mass spectrum in Eq. (3.22) and, for $\sigma=-1$, to set 4 of solutions in Eq. (3.22), as already derived. The control of the BBT sign factor $\sigma$, in Eq. (2.5), on the final solution structure appears here clearly. The profile solutions (3.22) have a custodian chiral structure as already described in Sec. II C.

Current from action variations.-In the presence of the BBTs (2.4) or (2.5) [invariant under the transformations (3.13)], as demonstrated in the beginning of Appendix B, the application of the Noether theorem based on the bulk EOM (3.3) - derived from the variation of the action (2.1)(2.3) invariant under the global $U(1)_{F}$ transformations (3.13) - leads to the same probability currents (B4) defined separately for the bulk fermions represented by the $5 \mathrm{D}$ fields $F=Q, D$, as in Eq. (3.12). Now, the NBCs (3.29) or (3.33) induced by the BBTs, as both satisfying the BCs (3.16), lead to four conditions of vanishing probability currents of the exact form (3.15). In other words, the presence of the BBTs guarantees (without imposing any condition) the vanishing of the currents at both boundaries for each independent fermion species. These BBT-induced conditions contribute to the consistent and complete definition of the geometrical field configuration for the considered model with a finite extra spatial interval in which fermionic matter is stuck.

Current from equations of motion.-Alternatively, we can derive directly (without the Noether theorem) the conservation relations, $\partial_{M} j_{F}^{M}=0$, for the probability currents (3.12) from a rewriting ${ }^{28}$ of each free 5D Dirac equation (3.3) in the bulk as in Eq. (3.24). The BBTs (2.4) or (2.5) affect only the NBCs derived from variation of the action (2.1)-(2.3). The NBCs (3.29) or (3.33), induced by the BBTs, both respect the BCs (3.16) and hence lead to the four conditions of vanishing currents (3.15). Therefore, as above, the BBTs guarantee the vanishing of the currents at

\footnotetext{
form.
} 
both boundaries for each fermion species. Those conditions allow a consistent and complete definition of the geometrical field setup for the model with matter on an interval.

Let us close this part by remarking that one could as well combine the two approaches to define the model: add a BBT only on an interval boundary for a given 5D field (as in this subsection) and apply the vanishing current condition only on the other boundary (as in Sec. III A 2).

\section{Presence of Yukawa couplings}

In this section, we only describe the two steps of a first method [29,35,60-67], that will turn out to be the correct method, for including the effects of the Yukawa terms (2.8) on the final fermion spectrum. First, the free profiles and free spectrum are calculated within a strict 5D approach whose correct treatment was exposed in details in Secs. III A 2 and III A 3. Second, one can write a mass matrix for the 4D fermion fields involving the pure $\mathrm{KK}$ masses (the free spectrum of the first step) as well as the masses induced by the Higgs VEV in the Yukawa terms (2.8) (with free profiles of the first step), which mix together the KK modes. The bidiagonalization of this matrix gives rise to an infinite set of eigenvalues constituting the physical masses, as will be presented in Sec. III B.

\section{B. 4D fermion mass matrix}

We focus on the fermion terms of the 5D action (2.9) in order to work out the mass spectrum: in particular on $S_{\Psi}, S_{X}$ and possibly $S_{B}$ [without direct effect on the mass matrix (3.35) as explained in Sec. IIC] if no EBCs are applied. Those terms lead-after insertion of the KK decomposition (3.5), use of free Eq. (3.7), orthonormalization condition (3.11), and integration over the fifth dimension - to the canonical kinetic terms for the 4D fermion fields as well as to the following fermionic 4D effective mass terms in the Lagrangian density (and to independent 4D effective Higgs-fermion couplings not discussed here),

$$
-\chi_{L}^{\dagger} \mathcal{M} \chi_{R}+\text { H.c. }
$$

in the combined basis for the left- and right-handed (transposed) 4D fields:

$$
\left\{\begin{array}{l}
\chi_{L}^{t}=\left(Q_{L}^{0 t}, Q_{L}^{1 t}, D_{L}^{1 t}, Q_{L}^{2 t}, D_{L}^{2 t}, \cdots\right) \\
\chi_{R}^{t}=\left(D_{R}^{0 t}, Q_{R}^{1 t}, D_{R}^{1 t}, Q_{R}^{2 t}, D_{R}^{2 t}, \cdots\right)
\end{array} .\right.
$$

Notice that there exists only one chirality for the zero modes as explained below Eq. (2.4). The infinite mass matrix reads as

$$
\mathcal{M}=\left(\begin{array}{cccccc}
\alpha_{00} & 0 & \alpha_{01} & 0 & \alpha_{02} & \cdots \\
\alpha_{10} & m_{1} & \alpha_{11} & 0 & \alpha_{12} & \cdots \\
0 & \beta_{11} & m_{1} & \beta_{12} & 0 & \cdots \\
\alpha_{20} & 0 & \alpha_{21} & m_{2} & \alpha_{22} & \cdots \\
0 & \beta_{21} & 0 & \beta_{22} & m_{2} & \cdots \\
\vdots & \vdots & \vdots & \vdots & \vdots & \ddots
\end{array}\right)
$$

where $m_{n}$ is the free spectrum (3.23). In this equation, the free wave function overlaps with the Higgs-brane are defined by (for real wave functions)

$$
\left\{\begin{array}{l}
\forall(i, j) \in \mathbb{N}^{2}, \alpha_{i j}=\frac{X}{L} q_{L}^{i}(L) d_{R}^{j}(L) \\
\forall(i, j) \in \mathbb{N}^{\star 2}, \beta_{i j}=\frac{X^{\prime}}{L} d_{L}^{i}(L) q_{R}^{j}(L)
\end{array} .\right.
$$

As the profiles are the free ones [profiles and KK mass spectrum solutions (3.21) with SM chiral structure], only the $\alpha_{i j}$ coefficients do not vanish.

The physical fermion mass spectrum is obtained by bidiagonalizing the mass matrix (3.35). This method is called the perturbation method in the sense that truncating the mass matrix at a given KK level corresponds to keeping only the dominant contributions to the lightest mass eigenvalue being the measured fermion mass (higher KK modes tend to mix less to the zero mode due to larger mass differences).

Extracting the mass spectrum equation from the characteristic equation for the Hermitian-squared mass matrix (3.35), in the case of infinite KK towers, is not trivial. This useful exercise was addressed analytically in Ref. [35] for the present toy model but with a 5D Yukawa coupling constant (and in turn a $X$ quantity) taken real. The resulting exact equation-without any approximation-was found to be

$\tan ^{2}\left(\sqrt{\left|M_{n}\right|^{2}} L\right)=X^{2} \Leftrightarrow \tan \left(\sqrt{\left|M_{n}\right|^{2}} L\right)= \pm X \quad(\forall n \geq 0)$.

Let us present here the absolute values of the solutions (physical masses) of this equation,

$$
\left|M_{n}\right|=\left|\frac{\arctan ( \pm X)+(-1)^{n} \tilde{n}(n) \pi}{L}\right| \quad(\forall n \geq 0),
$$

where the function $\tilde{n}(n)$ is defined by

$$
\left\{\begin{array}{l}
\tilde{n}(n)=\frac{n}{2} \text { for } n \text { even } \\
\tilde{n}(n)=\frac{n+1}{2} \text { for } n \text { odd }
\end{array}\right.
$$

so that the positive integer $n$ labeling the mass eigenvalues remains as well the label of the associated [as in the free case (3.6)] 4D mass eigenstates $\psi^{n}\left(x_{\mu}\right)$ [like in Eq. (3.5)]. 
Besides, globally speaking, for the whole set of $n$ levels, the absolute value of the fermion mass has a common generic expression for the two different classes $( \pm X)^{29}$ of solutions:

$$
\begin{aligned}
& \tan \left(\sqrt{\left|M_{n}\right|^{2}} L\right)=+X \\
& \Rightarrow\left|M_{n}\right|=\mid \frac{\arctan (X)+(-1)^{n} \tilde{n}(n) \pi}{L},
\end{aligned}
$$

$$
\begin{aligned}
\text { or, } & \tan \left(\sqrt{\left|M_{n}\right|^{2}} L\right)=-X \\
& \Rightarrow\left|M_{n}\right|=\frac{1}{L}\left|-\arctan (X)+(-1)^{n} \tilde{n}(n) \pi\right| \\
& =\frac{1}{L}\left|\arctan (X)+(-1)^{n+1} \tilde{n}(n) \pi\right| \\
& \equiv \frac{\arctan (X)+(-1)^{n} \tilde{n}(n) \pi}{L} .
\end{aligned}
$$

The last equality is justified for the whole spectrum by the fact that, for two consecutive $n$ values (for one odd $n$ and the following even $n$, with $\left.n>0^{30}\right),(-1)^{n}$ and $(-1)^{n+1}$ span the same two values \pm 1 while $\tilde{n}(n)$ keeps the same value. Hence, the two classes of solutions in Eqs. (3.40) and (3.41) may only differ by some different mass signs (remaining unfixed in the solutions for absolute values).

To check that the counting of states is correct, we observe that, in the realistic case $|X| \ll 1$ (typically small SM masses compared to the KK scale), two consecutive absolute masses $\left|M_{n}\right|$ (for one odd $n$ and the following even $n$, with $n>0$ ) of Eq. (3.38) are equal at leading order to the corresponding (unique $\tilde{n}$ value) absolute mass $| \pm \tilde{n} \pi / L|$ as in the free spectrum (3.23). Hence, in the vanishing mixing limit [see matrix (3.35)], the two associated consecutive mass eigenstates $\psi^{n}\left(x_{\mu}\right)$ tend well to the two free $4 \mathrm{D}$ field components $Q^{\tilde{n}}\left(x_{\mu}\right)$ and $D^{\tilde{n}}\left(x_{\mu}\right)$ [of Eq. (3.6)].

\section{5D TREATMENT: THE REGULARIZATION DOOM}

In this part, we work out the fermion mass spectrum in the defined model with the 5D action (2.9) using the alternative 5D approach based on the brane-Higgs regularization $[5,27,28,30,35,51,53]$, and we point out nonrigorous patterns of this method.

\footnotetext{
${ }^{29}$ The other solution, consisting alternatively of Eq. (3.40) for some $n$ levels and Eq. (3.41) for other $n$ levels, has to be ruled out since the complete and consistent infinite mass spectrum solution is fixed in a unique model hypothesis selected among the two given mathematical solutions (3.40) or (3.41), as Eq. (3.37) determines the quantity $\sqrt{\left|M_{n}\right|^{2}} L$ modulo $\pi$.

${ }^{30}$ The justification is obvious (no sign effect) for the special $n$-even case $\tilde{n}(0)=0$.
}

\section{A. Mixed Kaluza-Klein decomposition}

As we have just seen in Eqs. (3.34) and (3.35), after EW symmetry breaking, the infinite $Q_{L}^{n}$ and $D_{L}^{n}$ field towers mix together (as do the $Q_{R}^{n}$ and $D_{R}^{n}$ ) to form 4D fields $\psi_{L}^{n}$ (and $\psi_{R}^{n}$ ) representing mass eigenstates. To take into account this mixing within the 5D approach, these common 4D fields $\psi_{L}^{n}$ are defined instead of the $Q_{L}^{n}$ and $D_{L}^{n}$ fields (and similarly for the right-handed fields) in the whole KK decomposition, then called a mixed KK decomposition [instead of the free one in Eq. (3.5)] [28], as follows:

$$
\left\{\begin{array}{l}
Q_{L}\left(x_{\mu}, y\right)=\frac{1}{\sqrt{L}} \sum_{n=0}^{+\infty} q_{L}^{n}(y) \psi_{L}^{n}\left(x_{\mu}\right) \\
Q_{R}\left(x_{\mu}, y\right)=\frac{1}{\sqrt{L}} \sum_{n=0}^{+\infty} q_{R}^{n}(y) \psi_{R}^{n}\left(x_{\mu}\right) \\
D_{L}\left(x_{\mu}, y\right)=\frac{1}{\sqrt{L}} \sum_{n=0}^{+\infty} d_{L}^{n}(y) \psi_{L}^{n}\left(x_{\mu}\right) \\
D_{R}\left(x_{\mu}, y\right)=\frac{1}{\sqrt{L}} \sum_{n=0}^{+\infty} d_{R}^{n}(y) \psi_{R}^{n}\left(x_{\mu}\right)
\end{array} .\right.
$$

The 4D fields $\psi_{L / R}^{n}(\forall n)$ must satisfy the Weyl equations

$$
\left\{\begin{array}{l}
i \bar{\sigma}^{\mu} \partial_{\mu} \psi_{L}^{n}\left(x_{\mu}\right)=M_{n} \psi_{R}^{n}\left(x_{\mu}\right) \\
i \sigma^{\mu} \partial_{\mu} \psi_{R}^{n}\left(x_{\mu}\right)=M_{n} \psi_{L}^{n}\left(x_{\mu}\right)
\end{array},\right.
$$

where the spectrum $M_{n}$ includes the mass contribution whose origin is the Yukawa couplings (2.8). Note that, in contrast with the free case, there is a unique mass spectrum $M_{n}$ for a unique $4 \mathrm{D}$ field tower $\psi_{L / R}^{n}\left(x_{\mu}\right)$. To guarantee the existence of diagonal and canonical kinetic terms for those $4 \mathrm{D}$ fields $\psi_{L / R}^{n}$, the associated new profiles must now obey the two following orthonormalization conditions,

$\frac{1}{L} \int_{0}^{L} d y\left[q_{\mathcal{C}}^{n \star}(y) q_{\mathcal{C}}^{m}(y)+d_{\mathcal{C}}^{n \star}(y) d_{\mathcal{C}}^{m}(y)\right]=\delta_{n m}, \quad \forall n, \quad \forall m$,

for a chirality index $\mathcal{C} \equiv L$ or $R$. These two conditions are different from the four ones of Eq. (3.11) due to the new mixed KK decomposition.

\section{B. Inconsistencies of the Higgs shift procedure}

Here, we highlight the formal problems of the 5D process of shifting the brane-Higgs field [27,30,35] to get the fermion mass tower. Once more, the considered fermion terms of the 5D action (2.9) are $S_{\Psi}$ and $S_{X}$ (without $S_{B}$, which was missed in the relevant literature and which will be taken into account in Sec. V). The variations of the studied action lead to the same free BCs [second line of Eq. (3.2)] but to the following bulk EOM including the Yukawa coupling constants [instead of the free ones in Eq. (3.3)]: ${ }^{31}$

\footnotetext{
${ }^{31}$ In the subsections on Higgs regularizations, we use the same Lagrangians as in the present paper but the results and conventions from Ref. [35].
} 


$$
\left\{\begin{array}{l}
i \bar{\sigma}^{\mu} \partial_{\mu} Q_{L}+\partial_{4} Q_{R}+\delta(y-L) X D_{R}=0 \\
i \sigma^{\mu} \partial_{\mu} Q_{R}-\partial_{4} Q_{L}+\delta(y-L) X^{\prime} D_{L}=0 \\
i \bar{\sigma}^{\mu} \partial_{\mu} D_{L}+\partial_{4} D_{R}+\delta(y-L) X^{\prime} Q_{R}=0 \\
i \sigma^{\mu} \partial_{\mu} D_{R}-\partial_{4} D_{L}+\delta(y-L) X Q_{L}=0
\end{array}\right.
$$

Indeed, in view of regularizing the brane-Higgs field, the Yukawa interactions must be included in the bulk EOM [35] - as done in the literature. Inserting the mixed KK decomposition (4.1) in these 5D field EOM (4.4) allows us to factorize out the 4D fields, obeying the 4D Dirac equations (4.2), and obtain the profile equations for each excited mode [instead of the free ones in Eq. (3.7)]:

$$
\forall n,\left\{\begin{array}{l}
\partial_{4} q_{R}^{n}(y)+M_{n} q_{L}^{n}(y)=-\delta(y-L) X d_{R}^{n}(y), \\
\partial_{4} q_{L}^{n}(y)-M_{n} q_{R}^{n}(y)=\delta(y-L) X^{\prime} d_{L}^{n}(y), \\
\partial_{4} d_{R}^{n}(y)+M_{n} d_{L}^{n}(y)=-\delta(y-L) X^{\prime} q_{R}^{n}(y), \\
\partial_{4} d_{L}^{n}(y)-M_{n} d_{R}^{n}(y)=\delta(y-L) X q_{L}^{n}(y) .
\end{array}\right.
$$

Here, we underline a first mathematical issue of this usual approach: introducing $\delta(y-L)$ Dirac peaks ${ }^{32}$ in these profile equations leads to relations between distributions ${ }^{33}$ and functions which are thus not mathematically consistent $[37,38]$.

The apparent "ambiguity" noticed in the literature (context of a warped extra dimension) was that the Yukawa terms in Eq. (4.5) are present only at the $y=L$ boundary and might thus affect the fermion BCs. To avoid this question of a potential problem (like a field vagueness), a regularization of the brane-Higgs coupling was suggested. This regularization forces to keep the free fermion $\mathrm{BCs}$ in the presence of Yukawa interactions.

\section{Regularization I drawbacks}

In the first type of regularization applied in the literature $[5,27,35]$, called regularization I, the BCs are considered at the first level of the procedure to be injected in Eq. (4.5) [35]. The free BCs impose $d_{L}^{n}(L)=q_{R}^{n}(L)=0$ [see, respectively, the first and fourth solutions in Eq. (3.21)] so that Eq. (4.5) is supposed to become

$$
\forall n,\left\{\begin{array}{l}
\partial_{4} q_{R}^{n}(y)+M_{n} q_{L}^{n}(y)=-\delta(y-L) X d_{R}^{n}(y), \\
\partial_{4} q_{L}^{n}(y)-M_{n} q_{R}^{n}(y)=0, \\
\partial_{4} d_{R}^{n}(y)+M_{n} d_{L}^{n}(y)=0, \\
\partial_{4} d_{L}^{n}(y)-M_{n} d_{R}^{n}(y)=\delta(y-L) X q_{L}^{n}(y) .
\end{array}\right.
$$

At this level, we point out a second lack of strictness in the standard treatment; the two vanishing right-hand sides of Eq. (4.6) originate from the assumption that $0 \times \delta(0)=0$,

\footnotetext{
${ }^{32}$ Strictly speaking, a Dirac peak is a distribution although its historical name is "Dirac delta function."

${ }^{33}$ Also called "generalized functions" in mathematical analysis.
}

whereas the quantity $0 \times \delta(0)$ is rigorously undefined, ${ }^{34}$ which should forbid continuing this standard method. ${ }^{35} \mathrm{In}$ the next step of this method, the usual mathematical trick is to shift the brane-Higgs coupling from the brane at $y=L$ ( $\mathrm{TeV}$ brane in a warped framework) by an amount $\epsilon$ :

$$
\forall n,\left\{\begin{array}{l}
\partial_{4} q_{R}^{n}(y)+M_{n} q_{L}^{n}(y)=-\delta(y-[L-\epsilon]) X d_{R}^{n}(y), \\
\partial_{4} q_{L}^{n}(y)-M_{n} q_{R}^{n}(y)=0, \\
\partial_{4} d_{R}^{n}(y)+M_{n} d_{L}^{n}(y)=0, \\
\partial_{4} d_{L}^{n}(y)-M_{n} d_{R}^{n}(y)=\delta(y-[L-\epsilon]) X q_{L}^{n}(y) .
\end{array}\right.
$$

Then, the integration of the four relations of Eq. (4.7) over an infinitesimal range, tending to zero and centered at $y=L-\epsilon$, leads to ${ }^{36}$

$\forall n,\left\{\begin{array}{l}q_{R}^{n}\left([L-\epsilon]^{+}\right)-q_{R}^{n}\left([L-\epsilon]^{-}\right)=-X d_{R}^{n}(L-\epsilon), \\ q_{L}^{n}\left([L-\epsilon]^{+}\right)-q_{L}^{n}\left([L-\epsilon]^{-}\right)=0, \\ d_{R}^{n}\left([L-\epsilon]^{+}\right)-d_{R}^{n}\left([L-\epsilon]^{-}\right)=0, \\ d_{L}^{n}\left([L-\epsilon]^{+}\right)-d_{L}^{n}\left([L-\epsilon]^{-}\right)=X q_{L}^{n}(L-\epsilon) .\end{array}\right.$

Another inconsistency arising here in the regularization process is the following one. The first and fourth relations in Eq. (4.8) show that the wave functions $q_{R}^{n}(y)$ and $d_{L}^{n}(y)$ possess a discontinuity at $y=L-\epsilon$. Hence, the functions $\partial_{4} q_{R}^{n}(y)$ and $\partial_{4} d_{L}^{n}(y)$ are not defined at $y=L-\epsilon$. Two of the integrations performed on Eq. (4.7) to get Eq. (4.8) are thus not well defined. The fundamental theorem of analy$\operatorname{sis}^{37}[70]$ cannot be applied for functions undefined on the whole interval of integration. Let us express this problem in other terms; the functions $\partial_{4} q_{R}^{n}(y)$ and $\partial_{4} d_{L}^{n}(y)$ being not defined at $y=L$ (in the limit $\epsilon \rightarrow 0$ ), the last two terms of the starting 5D action (2.3) - defined along the interval $\mathcal{C}^{1} \equiv[0, L]$-are not well defined. ${ }^{38}$ Another definition problem appears in this regularization; the action (2.8) is

\footnotetext{
${ }^{34}$ This quantity corresponds also to an undefined product, namely, $0 \times \infty$, within the original simplified description [68] still used in physics textbooks (together with normalization conditions): $\delta(y-L) \equiv\left\{\begin{array}{cc}0 & \text { if } y \neq L \\ \infty & \text { if } y=L\end{array}\right.$.

${ }^{35}$ Such $\delta(0)$ divergences are automatically regulated-by the exchange of infinite towers of KK scalar modes-for a braneHiggs coupled to bulk scalar fields within a minimal supersymmetric scenario [69].

${ }^{36}$ The integration of Eq. (4.7) could also be performed over the interval $[L-\epsilon, L]$; this variant of the calculation, suggested in an Appendix of Ref. [27], represents in fact an equivalent regularization process leading to the same physical results and with identical mathematical inconsistencies.

${ }^{37}$ Let $(a, b) \in \mathbb{R}^{2}$ and $g$ be a continuous function on $[a, b]$; then, $g$ admits continuous primitives on $[a, b]$. Let $G$ be one of them; then, one has $\int_{a}^{b} d y g(y)=G(b)-G(a)$.

${ }^{38}$ From the current point of view, the conservation condition (B9) - involving in particular the 5D probability current component (5.16) — cannot be properly written at any point along the fifth dimension since $q_{R}^{n}(y)$ and $d_{L}^{n}(y)$ have discontinuities at $y=L$ so that derivatives in $\partial_{4} j^{4}$ are not well defined there.
} 
ill defined [37,38] since the Dirac peak $\delta(y-L)$ enters in particular as a factor of the profiles $q_{R}^{n}(y)$ and $d_{L}^{n}(y)$ being not continuous at $y=L$, as deduced from Eq. (4.8) -in the limit $\epsilon \rightarrow 0$-combined with the free BCs imposing $d_{R}^{n}(L) \neq 0, q_{L}^{n}(L) \neq 0$ [see, respectively, the first and fourth solutions in Eq. (3.21)]. ${ }^{39}$ Finally, the $q_{R}^{n}(y)$ and $d_{L}^{n}(y)$ jumps at $y=L$, obtained when regularizing the brane-Higgs coupling, conflict with the field continuity axiom of the invoked theory of variation calculus and hence with Hamilton's variational principle [36].

In the following steps of this regularization I, one solves Eq. (4.7) first in the interval $[0, L-\epsilon]$ (bulk EOM without Yukawa couplings) and applies the free BCs at $y=0$ on the obtained profiles. Then, one solves similarly Eq. (4.7) on $[L-\epsilon, L]$ before applying the jump and continuity conditions (4.8) at $y=L-\epsilon$ on the resulting profiles. The last step is to apply the free BCs at $y=L$ on these profiles and take the limit $\epsilon \rightarrow 0$ (to recover the studied brane-Higgs model) on the written BCs. The obtained $\mathrm{BCs}$ give rise to the equation whose solutions constitute the fermion mass spectrum:

$$
\tan \left(M_{n} L\right)=X \quad(\forall n) .
$$

The absolute value of the mass spectrum induced by this equation is exactly the same as the $4 \mathrm{D}$ approach result of Eqs. (3.40) and (3.41).

\section{Regularization II drawbacks}

Within regularization II [27,28,30,35], the Higgs coupling is first shifted in the bulk equations (4.5), which become

$$
\forall n, \quad\left\{\begin{array}{l}
\partial_{4} q_{R}^{n}(y)+M_{n} q_{L}^{n}(y)=-\delta(y-[L-\epsilon]) X d_{R}^{n}(y), \\
\partial_{4} q_{L}^{n}(y)-M_{n} q_{R}^{n}(y)=\delta(y-[L-\epsilon]) X^{\prime} d_{L}^{n}(y), \\
\partial_{4} d_{R}^{n}(y)+M_{n} d_{L}^{n}(y)=-\delta(y-[L-\epsilon]) X^{\prime} q_{R}^{n}(y), \\
\partial_{4} d_{L}^{n}(y)-M_{n} d_{R}^{n}(y)=\delta(y-[L-\epsilon]) X q_{L}^{n}(y) .
\end{array}\right.
$$

Integrating these four relations over an infinitesimal range centered at $y=L-\epsilon$ gives

$$
\forall n, \quad\left\{\begin{array}{l}
q_{R}^{n}\left([L-\epsilon]^{+}\right)-q_{R}^{n}\left([L-\epsilon]^{-}\right)=-X d_{R}^{n}(L-\epsilon), \\
q_{L}^{n}\left([L-\epsilon]^{+}\right)-q_{L}^{n}\left([L-\epsilon]^{-}\right)=X^{\prime} d_{L}^{n}(L-\epsilon), \\
d_{R}^{n}\left([L-\epsilon]^{+}\right)-d_{R}^{n}\left([L-\epsilon]^{-}\right)=-X^{\prime} q_{R}^{n}(L-\epsilon), \\
d_{L}^{n}\left([L-\epsilon]^{+}\right)-d_{L}^{n}\left([L-\epsilon]^{-}\right)=X q_{L}^{n}(L-\epsilon) .
\end{array}\right.
$$

\footnotetext{
${ }^{39}$ The profiles $q_{L}^{n}(y)$ and $d_{R}^{n}(y)$ are usually assumed to be continuous at $y=L-\epsilon$, while $q_{R}^{n}(y)$ and $d_{L}^{n}(y)$ remain unknown exactly at this point.
}

This set of conditions shows that the four wave functions undergo a jump at $y=L-\epsilon$ so that their derivatives with respect to $y$ are not defined at this point. Hence, the four integrations performed on Eq. (4.10) to obtain Eq. (4.11) are not well defined in this regularization. In other terms, the continuity conditions (4.11) rely on the right-hand sides of the equations so that one must choose a value for each profile exactly at $y=L-\epsilon$. Taking a standard mean value weighted thanks to a real number, $c$, Eq. (4.11) takes the form

$$
\forall n,\left\{\begin{array}{l}
q_{R}^{n}\left([L-\epsilon]^{+}\right)-q_{R}^{n}\left([L-\epsilon]^{-}\right)=-X \frac{d_{R}^{n}\left([L-\epsilon]^{-}\right)+c d_{R}^{n}\left([L-\epsilon]^{+}\right)}{1+c}, \\
q_{L}^{n}\left([L-\epsilon]^{+}\right)-q_{L}^{n}\left([L-\epsilon]^{-}\right)=X^{\prime} \frac{d_{L}^{n}\left([L-\epsilon]^{-}\right)+c d_{L}^{n}\left([L-\epsilon]^{+}\right)}{1+c}, \\
d_{R}^{n}\left([L-\epsilon]^{+}\right)-d_{R}^{n}\left([L-\epsilon]^{-}\right)=-X^{\prime} \frac{q_{R}^{n}\left([L-\epsilon]^{-}\right)+c q_{R}^{n}\left([L-\epsilon]^{+}\right)}{1+c}, \\
d_{L}^{n}\left([L-\epsilon]^{+}\right)-d_{L}^{n}\left([L-\epsilon]^{-}\right)=X^{q_{L}^{n}\left([L-\epsilon]^{-}\right)+c q_{L}^{n}\left([L-\epsilon]^{+}\right)} \\
1+c
\end{array}\right.
$$

Scrutinizing the left-hand sides of those four equations, one observes that jumps may arise at $y=L$ (under the limit $\epsilon \rightarrow 0$ ) for the four profiles (for each excited $n$th mode). Determining which profiles are discontinuous requires one to consider the free BCs at $y=L$ (before applying the limit $\epsilon \rightarrow 0$ ), the various $c$ values (including infinity), and the four profiles simultaneously [as they are related through Eq. (4.12)]. The hypothesis that all of the four profiles are continuous at $y=L-\epsilon$ (in the limit $\epsilon \rightarrow 0$ ) corresponds to the same field configuration as in the absence of Yukawa interactions $^{40}$ and leads thus to a free fermion mass spectrum. This kind of solution was not considered in the literature since it does not reproduce the SM at low energies and is thus not realistic. Therefore, there exists at least one profile discontinuous at $y=L$, which in turn cannot be derived at this point and leads to an undefined kinetic term [in the last two terms of 5D action (2.3)]. Furthermore, the obtained discontinuous [at $y=L$ ] profile comes in the factor of $\delta(y-L)$ in Eq. (2.8), spoiling the mathematical validity of this action. Besides, once more, this jump obtained at $y=L$ within the regularization process is not compatible with the field continuity axiom implicitly used when applying the Hamilton's variational principle.

In the next steps of regularization II, Eq. (4.10) is first solved over the domain $[0, L-\epsilon]$ (free bulk EOM) and the free $\mathrm{BC}$ at $y=0$ are applied on the resulting wave functions. Equation (4.10) is then solved over $[L-\epsilon, L]$ before the jump/continuity conditions (4.12) at $y=L-\epsilon$ are applied on the obtained profiles. Finally, the free BCs at $y=L$ are implemented on those profiles, and one applies

\footnotetext{
${ }^{40}$ Free BCs for continuous profiles and free version of the bulk equations (4.10) without the jump conditions (4.12) at $y=L-\epsilon$ involving effectively the Yukawa couplings.
} 
the limit $\epsilon \rightarrow 0$ on the expressed BCs. These BCs make the following fermion mass spectrum equation for $c=1$ appear:

$$
\tan \left(M_{n} L\right)=\frac{4 X}{4+X X^{\prime}} \quad[\forall n] .
$$

\section{Inconsistencies of the softened brane-Higgs coupling}

Another type of regularization used in the literature (on warped models) [27,28,30-33,35] consists in replacing the Dirac peak $\delta(y-L)$ of Eq. (2.8) by a normalized square function which has a vanishing width $(\epsilon)$ and an infinite value $(1 / \epsilon)$ in the limit $\epsilon \rightarrow 0$ where one expects to recover the considered model with a brane-Higgs coupling. Nevertheless, we point out here that the Dirac peak $\delta(y-L)$ at the Higgs brane, and in turn the original model, is not rigorously recovered via a limit, $\delta(y-L)=$ $\lim _{\epsilon \rightarrow 0} \eta_{\epsilon}(y-L)$, of a so-called nascent delta function (or delta sequence) $\eta_{\epsilon}$-here the mentioned square function-since such an equality is only symbolic; a distribution cannot be defined as the simple direct limit of a basic function. ${ }^{41}$ Hence, this would-be regularization is not satisfactory in the sense that it does not strictly reproduce the studied brane-Higgs scenario. By the way, notice that no profile jump is needed to be imposed in this regularization.

In addition, the two schemes of regularizations I and II still hold in this framework of a softened coupling, and in case of regularization I, a problem arises again: some terms of the profile EOM are taken at zero based on the assumption that $0 \times \delta(0)=0$, whereas the quantity $0 \times \delta(0)$ is undefined.

\section{Two noncommutativities of calculation steps}

The analytical differences of the mass spectra found in regularizations I and II, as well as via the softened and shifted brane-Higgs peaks, could be compensated by the different input values of the Yukawa coupling constant parameters $\left(Y_{5}\right.$ and $\left.Y_{5}^{\prime}\right)$ to get identical physical mass values. Nevertheless, regularizations I and II are in fact physically different. This difference is induced by the existence of measurable flavor violating effective 4D Yukawa couplings at leading order in $v^{2} / m_{1}^{2}$, which are generated by the $Y_{5}^{\prime}$ couplings [28] being present exclusively within regularization II (as appears clearly in the 4D approach). This physical difference between the two

\footnotetext{
${ }^{41}$ Strictly speaking, it is the effect of the Dirac peak in the integration of a function $f(y)$ over an interval covering the point $y=L, \int \delta(y-L) f(y) d y=f(L)$, which can be reproduced via an integration of the type, $\lim _{\epsilon \rightarrow 0} \int \eta_{\epsilon}(y-L) f(y) d y=f(L)$, not performed in the present regularization.
}

schemes of regularization raises the paradoxical question of which one is the sole correct analytical scheme to use and represents thus a confirmation of the inconsistency of regularizing the Higgs peak. These two schemes of regularization are obtained [35] by commuting in the 4D calculation (of masses and couplings) the ordering of implementation of the two limits $\epsilon \rightarrow 0$ [the regularizing parameter $\epsilon$ defined in Eq. (4.7)] and $N \rightarrow \infty$ [the upper value $N$ of the KK level $n$ in Eq. (3.5)]. Therefore, this physical noncommutativity of calculation steps reflects the inconsistency of the Higgs peak regularization. Another paradoxal noncommutativity of calculation steps arising in the context of regularization of a brane-Higgs coupled to bulk fermions was discussed in Refs. [31,32]: different results of Higgs production/decay rates when taking $\epsilon \rightarrow 0$ and then $N_{K K} \rightarrow \infty^{42}$ [30] or the inverse order [34] in their calculation. We can thus interpret now this second noncommutativity of calculation steps as being another effect, and in turn another confirmation, of the problematic Higgs regularization (also expected with a warped extra dimension). Hence, the theoretical debate in the literature about the origins of those two noncommutativities (involving $\epsilon$ ) finds its solution in the mathematically ill-defined (see above) and unnecessary (see below) Higgs regularization (introducing $\epsilon$ ).

\section{5D TREATMENT: THE CORRECT APPROACH}

In this part, we consider the presence of the Yukawa couplings (2.8) and present the rigorous 5D method to calculate the fermionic mass spectrum-which does not require any kind of regularization. We follow the main lines of the methodology developed for the free case in Sec. III A.

\section{A. Naive approach}

For the fermion masses, the relevant part of the considered action (2.9) to start with is

$$
S_{5 \mathrm{D}}^{\mathrm{m}}=S_{\Psi}+S_{X}-\int d^{4} x d y \delta(y) \mathcal{L}_{B}
$$

where the first term based on Eq. (2.3) can be recast into action (3.25) and $\mathcal{L}_{B}$ includes the BBTs of Eq. (2.4). Regarding the free brane at $y=0$, we could equivalently apply the EBCs (3.15) instead of including these BBTs, as we have exposed in details in Secs. III A 2 and III A 3. Now, without loss of generality, the least action principle leads to the four following conditions:

\footnotetext{
${ }^{42}$ Here, $N_{K K}$ stands for the number of excited fermion eigenstates exchanged at the loop level.
} 


$$
\begin{aligned}
& 0=\delta_{Q_{L}^{\dagger}} S_{5 \mathrm{D}}^{\mathrm{m}}=\int d^{4} x d y \delta Q_{L}^{\dagger}\left[i \bar{\sigma}^{\mu} \partial_{\mu} Q_{L}-\partial_{4} Q_{R}\right]+\left.\int d^{4} x\left[\delta Q_{L}^{\dagger}\left(\frac{1}{2} Q_{R}-X D_{R}\right)\right]\right|_{L}-\left.\int d^{4} x\left(\delta Q_{L}^{\dagger} Q_{R}\right)\right|_{0}, \\
& 0=\delta_{Q_{R}^{\dagger}} S_{5 \mathrm{D}}^{\mathrm{m}}=\int d^{4} x d y \delta Q_{R}^{\dagger}\left[i \sigma^{\mu} \partial_{\mu} Q_{R}+\partial_{4} Q_{L}\right]+\left.\int d^{4} x\left[-\delta Q_{R}^{\dagger}\left(\frac{1}{2} Q_{L}+X^{\prime} D_{L}\right)\right]\right|_{L}, \\
& 0=\delta_{D_{L}^{\dagger}} S_{5 \mathrm{D}}^{\mathrm{m}}=\int d^{4} x d y \delta D_{L}^{\dagger}\left[i \bar{\sigma}^{\mu} \partial_{\mu} D_{L}-\partial_{4} D_{R}\right]+\left.\int d^{4} x\left[\delta D_{L}^{\dagger}\left(\frac{1}{2} D_{R}-X^{\prime \star} Q_{R}\right)\right]\right|_{L}, \\
& 0=\delta_{D_{R}^{\dagger}} S_{5 \mathrm{D}}^{\mathrm{m}}=\int d^{4} x d y \delta D_{R}^{\dagger}\left[i \sigma^{\mu} \partial_{\mu} D_{R}+\partial_{4} D_{L}\right]+\left.\int d^{4} x\left[-\delta D_{R}^{\dagger}\left(\frac{1}{2} D_{L}+X^{\star} Q_{L}\right)\right]\right|_{L}+\left.\int d^{4} x\left(\delta D_{R}^{\dagger} D_{L}\right)\right|_{0} .
\end{aligned}
$$

Like in the studied free case, as the nonvanishing field variations $\delta F_{L / R}^{\dagger},\left.\delta F_{L / R}^{\dagger}\right|_{0, L}$ are generic, the sum of the first two terms (first line), in each of those four relations, must vanish separately, which brings in the same equations as the 5D EOM (3.3) and hence-via the mixed KK decomposition (4.1) and 4D Dirac-Weyl equations (4.2)-the profile equations

$$
\forall n \geq 0, \quad\left\{\begin{array}{l}
\partial_{4} q_{R}^{n}(y)-M_{n} q_{L}^{n}(y)=0 \\
\partial_{4} q_{L}^{n}(y)+M_{n} q_{R}^{n}(y)=0 \\
\partial_{4} d_{R}^{n}(y)-M_{n} d_{L}^{n}(y)=0 \\
\partial_{4} d_{L}^{n}(y)+M_{n} d_{R}^{n}(y)=0
\end{array}\right.
$$

whose solutions are found to be [with distinct constants for $f_{L / R}^{n}(y)=q_{L / R}^{n}(y)$ or $\left.f_{L / R}^{n}(y)=d_{L / R}^{n}(y)\right]$, as in Eq. (3.20),

$$
\begin{aligned}
& f_{L}^{n}(y)=B_{R}^{n} \cos \left(M_{n} y\right)-B_{L}^{n} \sin \left(M_{n} y\right), \\
& f_{R}^{n}(y)=B_{L}^{n} \cos \left(M_{n} y\right)+B_{R}^{n} \sin \left(M_{n} y\right) .
\end{aligned}
$$

The NBCs resulting from Eq. (5.2) read as

$$
\forall n \geq 0, \quad\left\{\begin{array}{l}
q_{R}^{n}(L)-2 X d_{R}^{n}(L)=0 \\
q_{L}^{n}(L)+2 X^{\prime} d_{L}^{n}(L)=0
\end{array}\right.
$$

$$
\begin{array}{rr}
d_{R}^{n}(L)-2 X^{\prime \star} q_{R}^{n}(L)=0, & q_{R}^{n}(0)=0 \\
d_{L}^{n}(L)+2 X^{\star} q_{L}^{n}(L)=0, & d_{L}^{n}(0)=0
\end{array}
$$

since the 4D fermion fields for the mass eigenstates cannot be linearly related-as discussed below Eq. (3.7). Those profile conditions, once applied on the solutions (5.4), lead to the form

$$
\forall n \geq 0,\left\{\begin{array}{l}
q_{L}^{n}(y)=C_{R}^{n} \cos \left(M_{n} y\right), \quad q_{R}^{n}(y)=C_{R}^{n} \sin \left(M_{n} y\right), \\
d_{L}^{n}(y)=-D_{L}^{n} \sin \left(M_{n} y\right), \quad d_{R}^{n}(y)=D_{L}^{n} \cos \left(M_{n} y\right),
\end{array}\right.
$$

together with the relations

$$
\left\{\begin{array}{lll}
\left.\left(Q_{R}-2 X D_{R}\right)\right|_{L}=0, & \left.\left(D_{R}-2 X^{\star \star} Q_{R}\right)\right|_{L}=0, & \left.Q_{R}\right|_{0}=0, \\
\left.\left(Q_{L}+2 X^{\prime} D_{L}\right)\right|_{L}=0, & \left.\left(D_{L}+2 X^{\star} Q_{L}\right)\right|_{L}=0, & \left.D_{L}\right|_{0}=0 .
\end{array}\right.
$$

Combining these NBCs leads to the consistency conditions on the Lagrangian parameters,

$$
4 X X^{\prime \star}=4 X^{\star} X^{\prime}=1,
$$

and in turn to $4\left|X X^{\prime}\right|=1$ and $\alpha_{Y^{\prime}}=\alpha_{Y}+2 k \pi$, where $k$ is an integer and $X=|X| e^{i \alpha_{Y}}$ and $X^{\prime}=\left|X^{\prime}\right| e^{i \alpha_{Y^{\prime}}}$ are the real numbers $\alpha_{Y}$ and $\alpha_{Y^{\prime}}$ representing the complex phases. The BCs (5.5), combined with the bulk profile EOM (5.3) [with solutions (5.4)] taken at $y=L$, constitute the complete BCs. Referring to the dependence on the quantity $X^{(\prime)}$, we denote $(X)$ this new class of complete BC at the brane with a Yukawa coupling (here at $y=L$ ) to distinguish them from the Dirichlet BCs usually noted (-) or the Neumann $\mathrm{BCs}$ noted $(+)$. The $\mathrm{BCs}(5.5)$ on the $5 \mathrm{D}$ fields give rise to the conditions on the profiles, through the KK decomposition (4.1),
These last two mass spectrum relations induced are strictly equivalent thanks to Eq. (5.6). The obtained mass spectrum allows us to determine, for instance, the BCs $(-\times)$ of the profile $d_{L}^{n}(y): d_{L}^{n}(0)=0$ and $d_{L}^{n}(L)=-D_{L}^{n} \sin \left(M_{n} L\right)$.

Let us check the validity of the obtained solutions. In the decoupling limit of high KK masses (compared to the 
typical SM energy scale) applied to the present model, one expects to recover approximately the SM setup at low energies. This decoupling condition is necessary for the theoretical consistency of the model, and it is generically imposed by the experimental constraints. First, according to Eq. (5.9), the lightest mode mass is

$$
\begin{aligned}
& M_{0}=\frac{1}{L} \arctan ( \pm 2|X|)=\frac{1}{L} \arctan \left( \pm \sqrt{2}\left|Y_{4} L v\right|\right) \\
& \underset{m_{1} \gg|v|}{\sim} \pm \sqrt{2}\left|Y_{4} v\right|,
\end{aligned}
$$

since $m_{1}=\pi / L$ [cf. Eq. (3.23)]. This 4D effective fermion mass [cf. Eq. (4.2)] is well proportional to the Higgs VEV as in the SM. Second, the effective Yukawa coupling constant in the 4D action term involving the lightest modes, $-\int d^{4} x Y_{00} H \psi_{L}^{0 \dagger} \psi_{R}^{0}+$ H.c., is obtained by injecting the KK decompositions (4.1) in Eq. (2.7) and then integrating over $y$, by using the wave functions (5.8) to take into account the mass mixings induced by the Yukawa couplings (5D method),

$$
\begin{aligned}
Y_{00} & =\frac{Y_{5}}{L} q_{L}^{0 \star}(L) d_{R}^{0}(L)+\frac{Y_{5}^{\prime \star}}{L} d_{L}^{0 \star}(L) q_{R}^{0}(L) \\
& =\frac{Y_{5}}{L} C_{R}^{0 \star} D_{L}^{0} \cos ^{2}\left(M_{0} L\right)-\frac{Y_{5}^{\prime \star}}{L} D_{L}^{0 \star} C_{R}^{0} \sin ^{2}\left(M_{0} L\right) \\
& =\frac{Y_{5}}{L} C_{R}^{0 \star} D_{L}^{0} \cos ^{2}\left(M_{0} L\right)-4 \frac{Y_{5}^{\prime \star}}{L} D_{L}^{0 \star} C_{R}^{0} X X^{\star} \cos ^{2}\left(M_{0} L\right) \\
& =\frac{Y_{5}}{L} C_{R}^{0 \star} D_{L}^{0} \cos ^{2}\left(M_{0} L\right)-4 \frac{Y_{5}^{\prime \star}}{L} \frac{\left(C_{R}^{0 \star}\right)^{2}}{D_{L}^{0 \star}} C_{R}^{0} X^{2} \cos ^{2}\left(M_{0} L\right) \\
& =\frac{Y_{5}}{L} C_{R}^{0 \star} \cos ^{2}\left(M_{0} L\right)\left[D_{L}^{0}-\frac{C_{R}^{0 \star}}{D_{L}^{0 \star}} C_{R}^{0}\right] \underset{m_{1} \gg|v|}{=} 0,
\end{aligned}
$$

where we have used subsequently the deduced equation and the relation involving $X^{\star}$ in the first line of Eq. (5.9) before invoking Eq. (5.6); as indicated right above, for high KK mass values, $C_{R}^{0} C_{R}^{0 \star}=D_{L}^{0} D_{L}^{0 \star}$, so that $Y_{00}$ vanishes, which differs from the SM framework. Indeed, applying the orthonormalization condition (4.3), for $n=m=0$, to the solution profiles (5.8), we deduce that

$$
\begin{array}{rl}
\int_{0}^{L} & d y\left[q_{L}^{0 \star}(y) q_{L}^{0}(y)+d_{L}^{0 \star}(y) d_{L}^{0}(y)\right] \\
\quad= & \int_{0}^{L} d y\left[q_{R}^{0 \star}(y) q_{R}^{0}(y)+d_{R}^{0 \star}(y) d_{R}^{0}(y)\right] \\
& \Leftrightarrow\left|C_{R}^{0}\right|^{2} \frac{\sin \left(2 M_{0} L\right)}{2 M_{0} L}=\left|D_{L}^{0}\right|^{2} \frac{\sin \left(2 M_{0} L\right)}{2 M_{0} L},
\end{array}
$$

which induces $\left|C_{R}^{0}\right|^{2}=\left|D_{L}^{0}\right|^{2}$ (and in turn $\left|D_{L}^{0}\right|^{2} \neq 0$ ) in the decoupling limit of Eq. (5.10) where $\left|2 M_{0} L\right| \underset{m_{1} \gg|v|}{\sim}$ $2 \sqrt{2}\left|Y_{4} v L\right| \ll 2 \sqrt{2}\left|Y_{4}\right| \pi=\mathcal{O}(\pi)$ and hence $\left|2 M_{0} L\right|<\pi$ so that we can divide Eq. (5.12) by $\sin \left(2 M_{0} L\right) / 2 M_{0} L$ being nonvanishing. ${ }^{43}$ The decoupling condition is thus not respected, which reveals a problem in the present treatment of the studied model. The problematic vanishing of the effective 4D Yukawa coupling constant $Y_{00}$ results from the invariance of the action (5.1) under the exchange transformation, $Q \leftrightarrow D$ together with $Y_{5}^{\star} \leftrightarrow Y_{5}^{\prime}$ at $y=L$ [symmetry also explicit in Eqs. (5.3) and (5.7)]; this symmetry will be broken in the correct treatments presented below. A confirmation of the failure of the present $5 \mathrm{D}$ treatment is the nonmatching of the obtained spectrum equation (5.9) with the 4D matrix method result (3.37). Therefore, the treatment of the brane-Higgs coupling of this subsection should be reconsidered; we present the other methods in the next two subsections.

\section{B. Introducing the fermion current condition (EBCs)}

Like in the free case treated in Sec. III A 2, we now try to define well the geometrical field configuration of the considered scenario based on the action $S_{5 \mathrm{D}}^{\mathrm{m}}$ of Eq. (5.1). In this scenario, the two 5D fields $Q$ and $D$ propagate only inside the interval $\mathcal{C}^{1} \equiv[0, L]$. This setup translates into a condition of vanishing probability current at both boundaries. The current is here the sum of the two individual currents of type (3.12) for the two species $Q$ and $D$ since those fermions are mixed together through the mass terms (2.8). To find out this current form rigorously, we first vary the action as in the beginning of Sec. VA and deduce the 5D EOM (3.3) whose profile solutions were given in Eq. (5.4). Then, using the obtained EOM (3.3), we apply in Appendix B the Noether theorem to work out the probability current (B9), ${ }^{44}$ which reads as

$$
j^{M}=-\alpha\left(\bar{Q} \Gamma^{M} Q+\bar{D} \Gamma^{M} D\right),
$$

as dictated by the global $U(1)$ symmetry of the action (5.1) relying on the transformations,

$$
Q_{L / R} \mapsto \mathrm{e}^{i \alpha} Q_{L / R}, \quad D_{L / R} \mapsto \mathrm{e}^{i \alpha} D_{L / R} .
$$

$\alpha(\in \mathbb{R})$ is a continuous parameter [now forced by the invariant terms (2.8) to be common for the two fields $Q, D$ ] involved, for example, in the infinitesimal field variations,

$$
\underline{\delta} Q_{L}=i \alpha Q_{L}, \quad \underline{\delta} Q_{L}^{\dagger}=-i \alpha Q_{L}^{\dagger} .
$$

We thus find that the effect of the Yukawa interactions is not to modify the currents but rather to force one to add them up for having a probability conservation relation (due to the induced mixing among the $Q$ and $D$ fields).

\footnotetext{
${ }^{43}$ We can also justify that $\sin \left(2 M_{0} L\right) / 2 M_{0} L$ is not vanishing from the deduced relation in the first line of Eq. (5.9) since one needs $|X| \neq 0$ to have $M_{0} \neq 0$ [cf. Eq. (5.10)] when $m_{1} \gg|v|$ (decoupling condition on the fermion mass).

${ }^{44}$ This result holds as well in the case without BBTs.
} 
Finally, the condition that the probability current vanishes (at the boundary where is located the Yukawa coupling) reads as ${ }^{45}$

$$
\begin{aligned}
\left.j^{4}\right|_{L} & =-\left.\alpha\left(\bar{Q} \Gamma^{4} Q+\bar{D} \Gamma^{4} D\right)\right|_{L} \\
& =\left.i \alpha\left(Q_{R}^{\dagger} Q_{L}-Q_{L}^{\dagger} Q_{R}+D_{R}^{\dagger} D_{L}-D_{L}^{\dagger} D_{R}\right)\right|_{L}=0 .
\end{aligned}
$$

For a nontrivial transformation with $\alpha \neq 0$, the field variation of this relation is

$$
\begin{aligned}
& \left(\delta Q_{R}^{\dagger} Q_{L}+Q_{R}^{\dagger} \delta Q_{L}-\delta Q_{L}^{\dagger} Q_{R}-Q_{L}^{\dagger} \delta Q_{R}+\delta D_{R}^{\dagger} D_{L}\right. \\
& \left.\quad+D_{R}^{\dagger} \delta D_{L}-\delta D_{L}^{\dagger} D_{R}-D_{L}^{\dagger} \delta D_{R}\right)\left.\right|_{L}=0 .
\end{aligned}
$$

The variation calculus chronology here is quite simple as no field is fixed by the EBCs (5.16): the fields (and their respective variations) are instead related via Eq. (5.16) [and Eq. (5.17)]. Now, the part of the variation of the action $S_{5 \mathrm{D}}^{\mathrm{m}}$, from Eq. (5.1), containing the boundary terms is written in Eq. (C1) of Appendix C. The complementary variation of the bulk action vanishing separately was already used just above to derive the 5D EOM (3.3). Notice that this variation of the bulk action with respect to the nonconjugate $5 \mathrm{D}$ fields in $\delta_{F_{L, R}} S_{5 \mathrm{D}}^{\mathrm{m}}$ requires an integration by parts to recover the Hermitian conjugate form of the EOM (3.3) [visible in Eq. (5.2)] and the boundary terms in $\delta_{F_{L, R}} S_{5 \mathrm{D}}^{\mathrm{m}}$ [visible in Eq. (C1)]. One could think of obtaining NBCs and their Hermitian conjugate form, respectively, from $\delta_{F_{L, R}} S_{5 \mathrm{D}}^{\mathrm{m}}$ and $\delta_{F_{L, R}^{\dagger}} S_{5 \mathrm{D}}^{\mathrm{m}}$ [as obtained in Eq. (5.5)], in Eq. (C1), but in fact, all the field variations are connected via the relation (5.17) so that one cannot get rid of those directly. There is no consistent way of combining the NBCs (C1) (even by splitting it into several vanishing expressions) with the EBCs (5.17) in order to get some set of BCs and another set made of the Hermitian conjugate BCs, except in the special but excluded case (see right below) in which

$$
\left.Q_{L}\right|_{L}=\left.D_{L}\right|_{L}=\left.Q_{R}\right|_{L}=\left.D_{R}\right|_{L}=0 .
$$

One could impose the condition $(\mathrm{C} 1)$ to be realized separately, leading to the NBCs (5.5) (and their Hermitian conjugate form) which induce ${ }^{46}$ the EBCs (5.16), but one would then be back to the case of Sec. VA, which has been ruled out due to the decoupling limit argue and the nonmatching of the $4 \mathrm{D}$ versus $5 \mathrm{D}$ results. The drastic BCs (5.18) (or the Hermitian conjugate form) imply obviously both the EBCs (5.16) and NBCs (C1) but lead to an inconsistency which reveals a problematic solution. Indeed, the BCs (5.18), once applied to the solutions (5.4), induce two equations that,

\footnotetext{
${ }^{45}$ The current condition at the other boundary is taken into account through the BBTs in the last term of Eq. (5.1).

${ }^{46} \mathrm{As}$ can be seen by replacing $\left.Q_{L, R}^{\dagger}\right|_{L}$ and $\left.D_{L, R}\right|_{L}$ in the expression (5.16) thanks to the two relations in the first and second columns of Eq. (5.5), respectively.
}

after being squared and summed together, give the identity $\left(B_{R}^{n}\right)^{2}=\left(B_{L}^{n}\right)^{2}[\forall n \geq 0]$ for both the $Q$ and $D$ fields: considering any $n$ level, the case $B_{R}^{n}= \pm B_{L}^{n}=0$ (for $Q$ and $D$ ) conflicts with the normalization condition (4.3), while for $B_{R}^{n}= \pm B_{L}^{n} \neq 0$ (for at least one of the two fields $Q$ and $D$ ), the two mentioned equations result in the simultaneous equalities $\cos \left(M_{n} L\right)=\sin \left(M_{n} L\right)$ and $\cos \left(M_{n} L\right)=$ $-\sin \left(M_{n} L\right)$ whose unique solution $\cos \left(M_{n} L\right)=$ $\sin \left(M_{n} L\right)=0$ makes no sense. As a conclusion, the impossibility to combine the EBCs and NBCs for getting some set of BCs together with a complementary set made of their Hermitian conjugate conflicts with the Feynman prescription for particles and antiparticles - according to which the fields and their Hermitian conjugate undergo identical physical equations (up to complex conjugate coupling constants). This conflict ${ }^{47}$ shows that the present approach of the configuration with a Yukawa coupling located at a boundary, based on the vanishing of the fermion current taken as an $\mathrm{EBC}$, is not yet the correct one. The origin of the problem is that the current (5.13) does not contain an explicit term that involves the Yukawa coupling constant.

\section{Introducing the bilinear brane terms (NBCs)}

As in the free case, we try here to apply the alternative treatment, based on the introduction of the BBTs at $y=L$, in order to develop a consistent approach. We consider the fermion part of the action (2.9),

$$
S_{5 \mathrm{D}}^{\prime \mathrm{m}}=S_{\Psi}+S_{B}+S_{X}
$$

based on the kinetic part (3.25), the BBTs (2.4), and the mass terms (2.8). The boundary fields $\left.F\right|_{0, L}$ are initially unknown so that their functional variations will be taken nonvanishing: $\left.\delta F\right|_{0, L} \neq 0$. Without loss of generality, the stationary action condition can be split into the two following conditions for each field $F=Q, D$ [extending Eqs. (3.27) and (3.28) to include the Yukawa terms] together with the two other equations $\delta_{F_{L}} S_{5 \mathrm{D}}^{\prime m}=\delta_{F_{R}} S_{5 \mathrm{D}}^{\prime m}=0$,

$$
\begin{aligned}
0=\delta_{F_{L}^{\dagger}} S_{5 \mathrm{D}}^{\prime \mathrm{m}}= & \int d^{4} x \int_{0}^{L} d y\left[\delta F_{L}^{\dagger} i \bar{\sigma}^{\mu} \partial_{\mu} F_{L}-\delta F_{L}^{\dagger} \partial_{4} F_{R}\right] \\
& +\int d^{4} x\left\{C_{F}^{L}\left[\left.\left(\delta F_{L}^{\dagger} F_{R}\right)\right|_{L}-\left.\left(\delta F_{L}^{\dagger} F_{R}\right)\right|_{0}\right]\right. \\
& \left.-\left.C_{F}^{L} X\left(\delta F_{L}^{\dagger} D_{R}\right)\right|_{L}-\left.C_{F}^{R} X^{\prime \star}\left(\delta F_{L}^{\dagger} Q_{R}\right)\right|_{L}\right\}
\end{aligned}
$$

\footnotetext{
${ }^{47}$ As described in the free case [below Eq. (3.25)], the direct injection of the EBC (5.16) in the action (5.1) would cancel out the boundary terms at $y=L$ in Eq. (3.25), spoiling then the needed Hermiticity of the whole action $S_{5 \mathrm{D}}^{\mathrm{m}}$ and leading thus to a related problem.
} 


$$
\begin{aligned}
0=\delta_{F_{R}^{\dagger}} S_{5 \mathrm{D}}^{\prime m}= & \int d^{4} x \int_{0}^{L} d y\left[\delta F_{R}^{\dagger} i \sigma^{\mu} \partial_{\mu} F_{R}+\delta F_{R}^{\dagger} \partial_{4} F_{L}\right] \\
& +\int d^{4} x\left\{C_{F}^{R}\left[-\left.\left(\delta F_{R}^{\dagger} F_{L}\right)\right|_{L}+\left.\left(\delta F_{R}^{\dagger} F_{L}\right)\right|_{0}\right]\right. \\
& \left.-\left.C_{F}^{R} X^{\star}\left(\delta F_{R}^{\dagger} Q_{L}\right)\right|_{L}-\left.C_{F}^{L} X^{\prime}\left(\delta F_{R}^{\dagger} D_{L}\right)\right|_{L}\right\},
\end{aligned}
$$

using the same $C_{Q, D}^{L, R}$ definitions as in Eqs. (3.27) and (3.28). Once more, the nonvanishing field variations $\delta F_{L / R}^{\dagger},\left.\delta F_{L / R}^{\dagger}\right|_{0, L}$ being generic, the sum of the first two terms (first line) in Eqs. (5.20) and (5.21), respectively, must vanish separately, which brings in the 5D EOM (3.3) and in turn - through the mixed KK decomposition (4.1) and 4D Dirac-Weyl equations (4.2) - the wave function equations (5.3) with solutions as in Eq. (5.4),

$$
\begin{aligned}
& q_{L}^{n}(y)=B_{R}^{n} \cos \left(M_{n} y\right)-B_{L}^{n} \sin \left(M_{n} y\right), \\
& q_{R}^{n}(y)=B_{L}^{n} \cos \left(M_{n} y\right)+B_{R}^{n} \sin \left(M_{n} y\right), \\
& d_{L}^{n}(y)=D_{R}^{n} \cos \left(M_{n} y\right)-D_{L}^{n} \sin \left(M_{n} y\right), \\
& d_{R}^{n}(y)=D_{L}^{n} \cos \left(M_{n} y\right)+D_{R}^{n} \sin \left(M_{n} y\right),
\end{aligned}
$$

using here new constant parameters $B_{L / R}^{n}, D_{L / R}^{n}$. Note that, in contrast, grouping directly the terms involving $C_{F}^{L / R}$ factors with the first two terms in Eqs. (5.20) and (5.21) — simply thanks to the introduction of Dirac peaks at $y=0, L$-would lead to mathematically meaningless relations between $5 \mathrm{D}$ functions and distributions. Finally, the NBCs resulting from Eqs. (5.20) and (5.21) read as

$$
\begin{aligned}
\left.\left(Q_{R}-X D_{R}\right)\right|_{L} & =0, & & \left.\left(D_{L}+X^{\star} Q_{L}\right)\right|_{L}=0, \\
\left.X^{\prime \star} Q_{R}\right|_{L} & =0, & & \left.X^{\prime} D_{L}\right|_{L}=0, \\
\left.Q_{R}\right|_{0} & =0, & & \left.D_{L}\right|_{0}=0 .
\end{aligned}
$$

Integrating by parts the bulk terms in the other relations $\delta_{F_{L}} S_{5 \mathrm{D}}^{\prime \mathrm{m}}=\delta_{F_{R}} S_{5 \mathrm{D}}^{\prime \mathrm{m}}=0$ allows us to recover the Hermitian conjugate form of the EOM (3.3) as well as the Hermitian conjugate form of the NBCs (5.23). The NBCs (5.23) can be rewritten without loss of generality as

$$
\begin{aligned}
\left.\left(Q_{R}-X D_{R}\right)\right|_{L} & =0,\left.\quad\left(D_{L}+X^{\star} Q_{L}\right)\right|_{L}=0, \quad X^{\prime}=0 \text { or } \\
\left\{\left.Q_{R}\right|_{L}\right. & \left.=0 \text { and }\left.D_{L}\right|_{L}=0\right\},\left.\quad Q_{R}\right|_{0}=0,\left.\quad D_{L}\right|_{0}=0,
\end{aligned}
$$

and in turn as

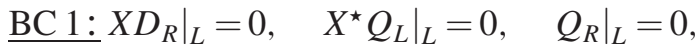

$$
\left.D_{L}\right|_{L}=0,\left.\quad Q_{R}\right|_{0}=0,\left.\quad D_{L}\right|_{0}=0,
$$

or,

$$
\begin{gathered}
\text { BC 2: }\left.\left(Q_{R}-X D_{R}\right)\right|_{L}=0,\left.\quad\left(D_{L}+X^{\star} Q_{L}\right)\right|_{L}=0, \\
X^{\prime}=0,\left.\quad Q_{R}\right|_{0}=0,\left.\quad D_{L}\right|_{0}=0 .
\end{gathered}
$$

The lightest fermionic state possesses a mass equal to the $\alpha_{00}$ element of the 4D mass matrix (3.35) in the decoupling limit $m_{1} \rightarrow \infty$ of the studied high-energy scenario, which allows us to reproduce well the SM mass expression at the lowenergy scales. For this purpose, one must have in particular a nonvanishing Yukawa coupling constant and $X \neq 0$ so that the $\mathrm{BC} 1$ reads as

$$
\begin{aligned}
& \text { BC 1: }\left.D_{R}\right|_{L}=0,\left.\quad Q_{L}\right|_{L}=0,\left.\quad Q_{R}\right|_{L}=0 \text {, } \\
& \left.D_{L}\right|_{L}=0,\left.\quad Q_{R}\right|_{0}=0,\left.\quad D_{L}\right|_{0}=0,
\end{aligned}
$$

where the BCs at $y=L$ are exactly similar to those in Eq. (5.18), which have already been ruled out. Hence, we exclude the $\mathrm{BC} 1$. Let us move to the $\mathrm{BC} 2$, which can be expressed in terms of the profiles, thanks to the relevant mixed KK decomposition (4.1), as follows (together with the condition $X^{\prime}=0$ ):

$$
\begin{aligned}
\text { BC 2: } & \forall n \geq 0, \quad q_{R}^{n}(L)-X d_{R}^{n}(L)=0, \\
& d_{L}^{n}(L)+X^{\star} q_{L}^{n}(L)=0, \quad q_{R}^{n}(0)=0, \quad d_{L}^{n}(0)=0 .
\end{aligned}
$$

So, these BC 2 at $y=0$ applied on the solutions (5.22) produce the following profiles:

$$
\begin{aligned}
& q_{L}^{n}(y)=B_{R}^{n} \cos \left(M_{n} y\right), \quad q_{R}^{n}(y)=B_{R}^{n} \sin \left(M_{n} y\right), \\
& d_{L}^{n}(y)=-D_{L}^{n} \sin \left(M_{n} y\right), \quad d_{R}^{n}(y)=D_{L}^{n} \cos \left(M_{n} y\right) .
\end{aligned}
$$

One must be careful to avoid some of the mathematical inconsistencies also encountered in the regularization procedures of Sec. IV: in particular, the existence of any profile jump at the interval boundaries, which would induce an undefined derivative term in the 5D action based on Eq. (2.3) (last two terms), an ill-defined term in the action (2.8) where the Dirac peak $\delta(y-L)$ would come in factor of a profile discontinuous at $y=L$-and finally would conflict with the field continuity axiom of the invoked theory of variation calculus. Therefore, we are taking all the profiles continuous at both boundaries, which is the reason why we have applied the BC 2 at $y=0$ on the bulk expressions (5.22). The application of the $\mathrm{BC} 2$ at $y=L$ on the resulting bulk expressions (5.25) gives rise to the relations (using $M_{n}, B_{R}^{n}, D_{L}^{n} \neq 0, \quad \forall n \geq 0$, to be checked a posteriori),

$$
\tan \left(M_{n} L\right)=X \frac{D_{L}^{n}}{B_{R}^{n}}=X^{\star} \frac{B_{R}^{n}}{D_{L}^{n}},
$$

which can be recast into (via $X D_{L}^{n} \hat{=}|X| D_{L}^{\prime n}, \forall n \geq 0$ )

$$
\tan \left(M_{n} L\right)= \pm|X|, \quad D_{L}^{\prime n}= \pm B_{R}^{n},
$$

assuming that the generic phase $\alpha_{Y}$ of the 5D Yukawa coupling constant, defined by $X=|X| e^{i \alpha_{Y}}$ as below Eq. (5.6), is included into a new parameter $D_{L}^{\prime n} \hat{=} D_{L}^{n} e^{i \alpha_{Y}}=$ $\left|D_{L}^{n}\right| e^{i\left(\alpha_{0}+\alpha_{Y}\right)}$. At this level, it is important to highlight the fact 
that it is the same \pm sign entering the two equalities in Eq. (5.26). We already remark the real mass spectrum resulting from Eq. (5.26), even for a Yukawa coupling constant with a nonvanishing imaginary part. Now, let us first apply the orthogonality conditions of Eq. (4.3) on the solutions (5.25):

$$
\begin{aligned}
& \int_{0}^{L} d y\left[D_{L}^{n \star} D_{L}^{m} \sin \left(M_{n} y\right) \sin \left(M_{m} y\right)\right. \\
& \left.\quad+B_{R}^{n \star} B_{R}^{m} \cos \left(M_{n} y\right) \cos \left(M_{m} y\right)\right]=0, \quad \forall n \neq m \\
& \int_{0}^{L} d y\left[B_{R}^{n \star} B_{R}^{m} \sin \left(M_{n} y\right) \sin \left(M_{m} y\right)\right. \\
& \left.\quad+D_{L}^{n \star} D_{L}^{m} \cos \left(M_{n} y\right) \cos \left(M_{m} y\right)\right]=0 .
\end{aligned}
$$

We insert trigonometric formulas ${ }^{48}$ in these equalities, in order to perform the integration, and then make use of another type of trigonometric relation ${ }^{49}$ to obtain the following simplified form $(\forall n, m$ with $n \neq m$ ):

$$
\begin{aligned}
& \frac{\sin \left(M_{m} L\right) \cos \left(M_{n} L\right)}{M_{n}^{2}-M_{m}^{2}}\left[M_{m} B_{R}^{n \star} B_{R}^{m}+M_{n} D_{L}^{n \star} D_{L}^{m}\right] \\
& =\left[M_{n} B_{R}^{n \star} B_{R}^{m}+M_{m} D_{L}^{n \star} D_{L}^{m}\right] \frac{\sin \left(M_{n} L\right) \cos \left(M_{m} L\right)}{M_{n}^{2}-M_{m}^{2}}, \\
& \frac{\sin \left(M_{m} L\right) \cos \left(M_{n} L\right)}{M_{n}^{2}-M_{m}^{2}}\left[M_{m} D_{L}^{n \star} D_{L}^{m}+M_{n} B_{R}^{n \star} B_{R}^{m}\right] \\
& =\left[M_{n} D_{L}^{n \star} D_{L}^{m}+M_{m} B_{R}^{n \star} B_{R}^{m}\right] \frac{\sin \left(M_{n} L\right) \cos \left(M_{m} L\right)}{M_{n}^{2}-M_{m}^{2}} .
\end{aligned}
$$

We can divide these equalities by $\cos \left(M_{n} L\right) \cos \left(M_{m} L\right)$ since $\cos \left(M_{n} L\right) \neq 0[\forall n]$ [as the mass spectrum given by Eq. (5.26) is not a free one]; we get $(\forall n, m$ with $n \neq m$ )

$$
\begin{aligned}
& \tan \left(M_{m} L\right)\left[M_{m} B_{R}^{n \star} B_{R}^{m}+M_{n} D_{L}^{n \star} D_{L}^{m}\right] \\
& \quad=\tan \left(M_{n} L\right)\left[M_{n} B_{R}^{n \star} B_{R}^{m}+M_{m} D_{L}^{n \star} D_{L}^{m}\right], \\
& \tan \left(M_{m} L\right)\left[M_{m} D_{L}^{n \star} D_{L}^{m}+M_{n} B_{R}^{n \star} B_{R}^{m}\right] \\
& \quad=\tan \left(M_{n} L\right)\left[M_{n} D_{L}^{n \star} D_{L}^{m}+M_{m} B_{R}^{n \star} B_{R}^{m}\right] .
\end{aligned}
$$

Using the spectrum of Eq. (5.26), getting rid of the common factor $\left(M_{n}-M_{m}\right)$ in those two equations and dividing the resulting equalities by the constant parameters allowing us to separate the $n$ and $m$ dependences, we find the unique relation

$$
\frac{D_{L}^{n \star}}{B_{R}^{n \star}}=\frac{B_{R}^{m}}{D_{L}^{m}}, \quad \forall n, m \quad \text { with } \quad n \neq m,
$$

which is clearly true since we know from Eq. (5.26) that $B_{R}^{n}= \pm D_{L}^{\prime n}= \pm D_{L}^{n} e^{i \alpha_{Y}}(\forall n)$. Second, we apply the $\mathrm{n}$

\footnotetext{
${ }^{48}$ Of the kind, $\cos \left(M_{n} y\right) \cos \left(M_{m} y\right)=\left[\cos \left(M_{n} y+M_{m} y\right)+\right.$ $\left.\cos _{49}\left(M_{n} y-M_{m} y\right)\right] / 2$.

${ }^{49} \sin \left(M_{n} L-M_{m} L\right)=\sin \left(M_{n} L\right) \cos \left(M_{m} L\right)-\cos \left(M_{n} L\right) \times$ $\sin \left(M_{m} L\right)$.
}

ormalization conditions of Eq. (4.3) on the profile solutions (5.25) obeying the constraints (5.26) (implying $\left.\left|B_{R}^{n}\right|^{2}=\left|D_{L}^{n}\right|^{2}\right)$

$$
\int_{0}^{L} d y\left|B_{R}^{n}\right|^{2}\left[\sin ^{2}\left(M_{n} y\right)+\cos ^{2}\left(M_{n} y\right)\right]=L, \quad \forall n .
$$

Those conditions allow us to complete Eq. (5.26), which becomes

$$
\forall n, \tan \left(M_{n} L\right)= \pm|X|, \quad D_{L}^{\prime n}= \pm B_{R}^{n}, \quad\left|B_{R}^{n}\right|=\left|D_{L}^{n}\right|=1
$$

and exhibits then the three types of solutions

$$
\begin{aligned}
& \text { I: } \forall n, \tan \left(M_{n} L\right)=+|X|, \quad B_{R}^{n}=e^{i\left(\alpha_{0}+\alpha_{Y}\right)}, \quad D_{L}^{n}=e^{i \alpha_{0}}, \\
& \text { II }: \forall n, \tan \left(M_{n} L\right)=-|X|, \quad B_{R}^{n}=e^{i\left(\alpha_{0}+\alpha_{Y} \pm \pi\right)}, \quad D_{L}^{n}=e^{i \alpha_{0}}, \\
& \text { III : solution I for some } n \text { values and II for other } n \text { values, }
\end{aligned}
$$

$\alpha_{0}$ becoming the common phase (defined modulo $2 \pi$ ). Following a similar discussion as the one below Eqs. (3.40) and (3.41), we can claim that for the whole set of $n$ levels the absolute value of the fermion mass has the following common expression for solutions I and II,

$$
\begin{aligned}
\tan \left(M_{n} L\right) & =+|X| \\
\Rightarrow\left|M_{n}\right| & =\frac{\arctan (|X|)+(-1)^{n} \tilde{n}(n) \pi}{L},
\end{aligned}
$$

or, $\tan \left(M_{n} L\right)=-|X|$

$$
\Rightarrow\left|M_{n}\right| \equiv \frac{\arctan (|X|)+(-1)^{n} \tilde{n}(n) \pi}{L}, \text {, }
$$

using the $\tilde{n}(n)$ function already defined in Eq. (3.39). Once again, solution III must be excluded as the complete and consistent infinite mass spectrum solution is determined within a unique model hypothesis selected among the two given mathematical solutions, I or II, since Eq. (5.29) fixes the quantity $M_{n} L$ modulo $\pi$.

Within the simplified case of a real 5D Yukawa coupling constant $(|X|=X)$, we thus find that the unique tower (5.29) of absolute values of the physical fermion masses is matching the one obtained in the 4D approach: Eqs. (3.40) and (3.41). This exact 4D-5D matching confirms the overall consistency of our calculations-without regularizations - and is, of course, expected to be reached as well for a complex 5D Yukawa coupling constant.

In particular, the insensitivity of the $4 \mathrm{D}$ fermion mass matrix (3.35) to the $Y_{5}^{\prime}$ coupling constant [described below Eq. (3.36)] matches interestingly the spectrum independence on $Y_{5}^{\prime}$ induced by the result $Y_{5}^{\prime}=0$ obtained in the BC 2 [see Eq. (5.24)] used for the 5D point of view. 
Let us give an intuitive interpretation of the absence of the role for the $Y_{5}^{\prime}$ coupling (involved in $X^{\prime}$ ) in the final spectrum (5.29) which depends only on the $X$ quantity. Starting with the free action $S_{\Psi}+S_{B}$, the profiles $d_{L}^{n}(y)$ and $q_{R}^{n}(y)[\forall n]$, defined by Eq. (3.5) and with solutions (3.21), vanish in particular at the boundary $y=L$. Hence, the term with a $X^{\prime}$ coefficient in the action piece $S_{X}$ of Eq. (2.8), once added to $S_{\Psi}+S_{B}$, is expected to have a vanishing factor coming from the integration over the interval due to the Dirac peak. This argument is only intuitive as it does not really include the possible "backreaction" effect of the $X^{\prime}$ term on the profiles via modified BCs.

Finally, let us discuss the condition on the fermion current for the boundary at $y=L$. Inserting the four expressions of $Q_{R}^{(\dagger)}$ and $Q_{L}^{(\dagger)}$, provided by the first two NBCs of Eq. (5.24) at $y=L$, in the current condition (5.16) leads to a trivially true equality (all the four terms involving exclusively $D_{L, R}^{(\dagger)}$ fields cancel each other). This feature means that the NBCs (5.24) imply the condition (5.16) so that the geometrical field setup of the present model with matter stuck on an interval is well defined.

As a conclusion, adding BBTs at the brane with the Yukawa coupling to bulk fermions permits a consistent treatment of the considered scenario and a correct calculation of the mass spectrum.

\section{OVERVIEW AND IMPLICATIONS}

\section{A. Action content}

In Table I, we summarize the results for the obtained fermion $\mathrm{BCs}$ at a single 3-brane. We conclude from this table that for fermions on an interval and coupled or not to a brane-localized Higgs field either BBTs should be generated in the action or conditions should arise on the fermion current (forcing then the 4D treatment in case of a brane Yukawa coupling) depending on the origin of the model at high energies. In the same spirit, notice that the UV completion will determine whether the selection of fermion boundary conditions is imposed or deduced from

TABLE I. Bulk fermion BCs (when a consistent determination exists) at a 3-brane where is located the Higgs boson coupled to bulk fermions, within the different cases of the three following boundary treatments: presence of BBTs, vanishing of the probability current, or none of those two situations. The 4D line holds as well for the 5D approach of the free brane. As usual, the Dirichlet BCs are noted (-), the Neumann BCs are noted $(+)$, and we denote $(\times)$ the new BCs depending on the Yukawa coupling constant.

\begin{tabular}{lccc}
\hline \hline & $\begin{array}{c}\text { No boundary } \\
\text { characteristic }\end{array}$ & $\begin{array}{c}\text { current condition } \\
(\mathrm{EBC})\end{array}$ & $\begin{array}{c}\text { Bilinear } \\
\text { brane terms } \\
(\mathrm{NBC})\end{array}$ \\
\hline 4D approach & $\begin{array}{c}(\text { Impossible }) \\
\text { 5D approach }\end{array}$ & $\begin{array}{c}\mathrm{BC}( \pm) \\
(\text { Impossible })\end{array}$ & $\mathrm{BC}( \pm)$ \\
\hline \hline
\end{tabular}

Higher-dimensional model definition

\section{1}

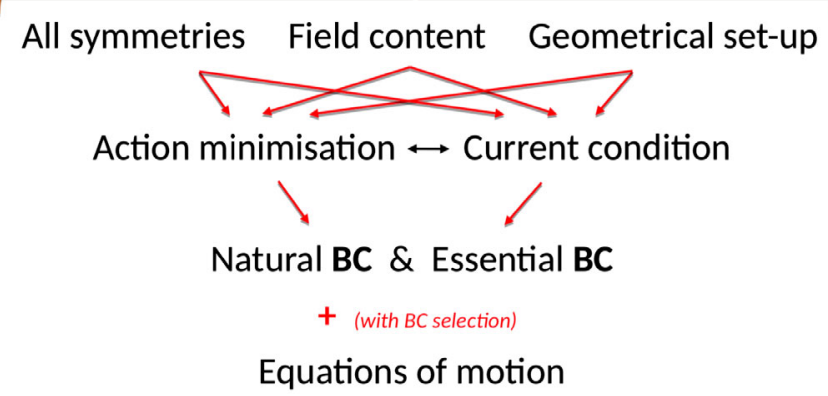

ת

\section{Mass spectrum,}

Profiles

FIG. 1. Inverse pyramidal picture illustrating the general principles for determining the wave functions and masses of mixed KK modes within a given model based on extra dimension (s). The same notations as in the main text are used.

the action form. The UV completion should not bring simultaneously EBCs (imposing vanishing currents) and BBTs (guaranteeing current vanishing) because it would be possible but redundant. It is interesting to observe anyway that the necessary additional fermionic ingredient, with respect to the kinetic terms, reveals that limiting the integration domain of the action does not suffice to define consistently and completely the basic field configuration along the interval (or more generally over a compactified space). Indeed, without having a vanishing fermion current at a boundary, one could imagine a source of creation or a mechanism of absorption for particles at the boundary. Therefore, the present status, resulting from this analysis and its synthesis, is that the action expression may not contain all the information (e.g., current conditions) needed to define a higher-dimensional model, regarding the geometrical setup and field configurations.

Based on the above results, we describe now the generic methodology to find out the mass spectrum and KK wave functions (allowing us to calculate the 4D effective couplings) along the extra spatial dimension(s) of a given scenario. For this purpose, we present in Fig. 1 a schematic description of the main principles. The figure must be understood as follows. A given extra-dimensional model must be first defined by its geometrical setup (space-time structure and field location configuration), its field content, and its internal symmetries (gauge groups,...) as well as other types of symmetries (the Poincaré group here) of symmetries. These three types of information determine entirely the action form ${ }^{50}$ whose minimization gives rise to

\footnotetext{
${ }^{50}$ Within a well-understood scenario, all terms of the Lagrangian density should be deduced from the model definition exclusively: the absence of couplings from symmetries, presence of BBTs from the geometrical setup, etc.
} 
the 5D EOM and NBCs. Besides, the geometrical hypotheses of the model, concerning for instance the space limits for field propagation, may induce probability current conditions translated into some EBCs, ${ }^{51}$ which must be combined with the above NBCs. At this level, a choice of the combined BCs obtained can be required (if not determined automatically by the action structure itself). Then, the KK decomposition (together with the EOM on the $4 \mathrm{D}$ fields) allows us to derive the EOM and BCs on the $\mathrm{KK}$ profiles along the extra dimension(s). The last step is obviously to solve these profile EOM, coupled to the complete $\mathrm{BCs}$, in order to work out the mass spectra.

\section{B. Implementation of the cutoff on energy}

We have to discuss the cutoff treatment as the framework of higher-dimensional models is nonrenormalizable theories, which are valid in a limited domain of energy, up to a certain scale, set by perturbativity conditions on effective dimensionless couplings. If the UV completion of such models affects the KK excitation towers and thus the fermion mass spectrum, in an unknown way, then its calculation must include the KK state masses only up to the cutoff value typically (the UV corrections at low energies can be parametrized via higher-dimensional operators). In a case of the absence of UV effects on the specific mass spectrum sector, the whole KK towers should be taken into account at the mass calculation level since even the smallest mass eigenvalues can be affected by the mixing effects of the infinite towers. Now, in both situations, only the eigenstates with masses up to the cutoff scale should be considered for the phenomenological observables (reaction amplitudes, rates, etc.) due to the nonrenormalizable nature of the theory. Technically, the implementation of an energy cutoff in the bulk fermion mass calculation and tree-level Lagrangian construction forces the use of the 4D approach. Indeed, the mixed KK decomposition (4.1), used in the 5D approach, includes the mixing of the whole tower; the fields $\psi_{L, R}^{n}\left(x_{\mu}\right)$ are mass eigenstates.

\section{Phenomenological impacts}

In the appropriate treatment developed in the present paper, without regularization, the obtained mass spectrum and effective 4D Yukawa coupling depend on $Y_{5}$ but not on the $Y_{5}^{\prime}$ coupling constant. For instance, in Eq. (5.11), one should in fact apply the result $Y_{5}^{\prime}=0$ as dictated by the relevant BC 2 in Eq. (5.24). Applying an energy cutoff in the process of mass calculation would not affect this independence on $Y_{5}^{\prime}$ as is clear from the point of view of the 4D approach.

The results for fermion masses and profiles are also correct when one invokes the Higgs peak regularization I, which

\footnotetext{
${ }^{51}$ The EBC could also originate from the definition of the symmetry of orbifold scenarios.
}

cancels out the $Y_{5}^{\prime}$ dependence. Hence, the phenomenological analyses of the literature based on such results are still valid; see, for instance, Refs. [5,7,8,29,67,71-74]. Those studies apply on the geometrical background with warped extra dimensions where the KK spectrum independence on $Y_{5}^{\prime}$ is expected to occur as well.

Note that the results from regularization I and the correct ones, in the approach without regularization at all, are exactly identical only by accident. Indeed, in regularization I, considering first the $5 \mathrm{D}$ treatment, the mass spectrum calculation in presence of Yukawa couplings suffers from two errors, which exactly cancel out each other; there are no BBTs, which affects the resulting spectrum equation by a factor 2 [as seen when comparing the spectra with BBTs in Eq. (5.29) and without BBTs in Eq. (5.9)], and a regularization is applied. Now, starting from the 4D treatment of regularization I and adding BBTs (or current conditions) would have no effect on the 4D mass matrix (as described in Sec. II C) like avoiding the regularization process (as there is no analytical effect of regularization I in which the limit $\epsilon \rightarrow 0$ is taken at the first step [35]); the results of regularization I are thus the same as in the correct approach.

In contrast, if the Higgs peak regularization II is used, the obtained fermion masses and 4D Yukawa couplings depend on both $Y_{5}$ and $Y_{5}^{\prime}$ so that the results differ effectively from the correct ones. Hence, the phenomenological studies based on these analytical results (for example, Refs. [28,30-33]) should be reconsidered.

For example, the effective 4D Yukawa couplings to fermions and their KK excitations affect the main Higgs production mechanism at the LHC: the gluon-gluon fusion via triangular loops of (KK) fermions. Hence, the effect of the realistic limit [35] of vanishing $Y_{5}^{\prime}$ on the constraints on KK masses derived in the studies [30-33], within the warped background and based on regularization II, should be calculated precisely.

Besides, the rotation matrices diagonalizing the 4D fermion mass matrix (3.35) do not diagonalize simultaneously the effective 4D Yukawa coupling matrix since the last one does not contain matrix elements made of the pure KK masses. The induced flavor violating 4D Yukawa couplings are generated at leading order by $Y_{5}^{\prime}$ contributions as can be shown diagrammatically [28]. Hence, there exist large flavor changing neutral current (FCNC) effects in measured $\Delta F=2$ processes such as $\bar{K}-K, \bar{B}-B$, and $\bar{D}-D$ mixings, mainly produced by tree-level exchanges of the Higgs boson via $Y_{5}^{\prime}$ couplings, which lead to considerable lower bounds on the KK boson mass scale (in balance via opposite Yukawa coupling dependences with the ones from the tree-level contribution of the KK gluon exchange) found to be around 6-9 $\mathrm{TeV}$ in the analysis [28] on warped extra dimensions using indeed regularization II. Hence, these bounds should be significantly suppressed in the realistic situation in which $Y_{5}^{\prime} \rightarrow 0$; this limit should indeed be applied since the independence 
found in the present paper upon $Y_{5}^{\prime}$ (extended via flavor indices) remains true for the case of three flavors, as well as for fermion bulk masses, as is clear in the 4D approach in which the $\beta_{i j}$ elements (3.36) of the mass matrix still vanish. The predictions of Ref. [28], based on regularization II, that FCNC reactions involving Yukawa couplings, like the rare top quark decay $t \rightarrow c h$ and exotic Higgs boson decay to charged leptons $h \rightarrow \mu \tau$, can be observable at the LHC deserve reconsiderations as well when $Y_{5}^{\prime}=0$.

\section{CONCLUSIONS}

For bulk fermions coupled to a brane-Higgs boson, we have shown that the proper calculation of the fermion masses and effective 4D Yukawa couplings does not rely on Higgs peak regularizations. The justifications are the following ones:

(i) There are no fermion wave function jumps at the Higgs boundary and so no motivation to introduce an arbitrary regularization.

(ii) The regularizations suffer from several mathematical discrepancies confirmed by two known noncommutativities of calculation steps.

(iii) The right method without any regularization is validated in particular by the converging results of the $4 \mathrm{D}$ versus $5 \mathrm{D}$ treatments.

In the rigorous method developed for both free and brane-coupled bulk fermions, we have also pointed out the necessity to either include BBTs in the Lagrangian density or alternatively impose vanishing conditions for probability currents at the interval boundaries. Here, the arguments go as follows:

(i) The presence of BBTs guarantees the vanishing current conditions, which define the field geometrical configuration of the model.

(ii) The BBT and current conditions allow us to find physically consistent fermion masses, bulk profiles, and effective 4D Yukawa couplings (solutions fulfilling the normalization constraints, the Hermitian conjugate $\mathrm{BCs}$, and the decoupling limit condition).

(iii) The BBTs lead to the expected matching between the $4 \mathrm{D}$ and $5 \mathrm{D}$ calculation results.

The BBTs represent a possible origin of the chiral nature of the SM as well as of its chirality distribution among quark/lepton $S U(2)_{L}$ doublets and singlets. Those terms could thus provide new clues about the UV completion of the SM.

Depending on the UV completion, the general methodology worked out reveals that the information regarding the definition of an higher-dimensional model are not necessarily fully contained in the action itself-through the deduced EOM and NBCs - but might be partly included as well in the EBCs.

We have finished the analysis by the descriptions of the appropriate energy cutoff procedure in the present framework and of the phenomenological impacts of the new calculation method which predicts the independence of the fermion masses and effective 4D Yukawa couplings on the $Y_{5}^{\prime}$ parameter of the Lagrangian. This different coupling feature, with respect to regularization II usually applied in the literature, should, in particular, suppress significantly the previously obtained severe bounds on KK masses induced by FCNC processes generated via flavor violating couplings of the Higgs boson.

An extension of the present study, to generic BBTs, fermion bulk masses, warped extra dimensions, and orbifold scenarios, is under progress [49].

\section{ACKNOWLEDGMENTS}

The authors thank Emilian Dudas, Ulrich Ellwanger, Adam Falkowski, Maria Kitsara, and Jérémie Quevillon for useful discussions. R. L. is supported by the agreement signed between the China Scholarship Council and the University Paris-Sud. F. N. acknowledges support from the IDEX Paris-Saclay. G. M. and F. N. are grateful to the "Commission du collège doctoral" of the University Paris-Saclay as well as to the University Paris-Sud for the complementary funding.

\section{APPENDIX A: NOTATIONS AND CONVENTIONS}

Throughout the present paper, we use the conventions of the Ref. [75]. The 5D Minkowski metric is

$$
\eta_{M N}=\operatorname{diag}(+1,-1,-1,-1,-1) .
$$

The 4D Dirac matrices are taken in the Weyl representation,

$$
\gamma^{\mu}=\left(\begin{array}{cc}
0 & \sigma^{\mu} \\
\bar{\sigma}^{\mu} & 0
\end{array}\right) \text { with }\left\{\begin{array}{l}
\sigma^{\mu}=\left(\mathbb{I}, \sigma^{i}\right), \\
\bar{\sigma}^{\mu}=\left(\mathbb{I},-\sigma^{i}\right),
\end{array}\right.
$$

where $\sigma^{i}(i=1,2,3)$ are the three Pauli matrices:

$\sigma^{1}=\left(\begin{array}{ll}0 & 1 \\ 1 & 0\end{array}\right), \quad \sigma^{2}=\left(\begin{array}{cc}0 & -i \\ i & 0\end{array}\right), \quad \sigma^{3}=\left(\begin{array}{cc}1 & 0 \\ 0 & -1\end{array}\right)$.

One has also the 4D chirality operator

$$
\gamma^{5}=i \prod_{\mu=0}^{3} \gamma^{\mu}=\left(\begin{array}{cc}
-\mathbb{I} & 0 \\
0 & \mathbb{I}
\end{array}\right)
$$

With our conventions, the 5D Dirac matrices read as

$$
\Gamma^{M}=\left(\gamma^{\mu}, i \gamma^{5}\right)
$$




\section{APPENDIX B: NOETHER THEOREM INCLUDING BRANE-LOCALIZED TERMS}

Here, we demonstrate the Noether theorem in the presence of boundary-localized Yukawa couplings and BBTs. We first consider the free action (2.1) together with the BBTs (2.4) [or (2.5)] being invariant under the transformations (3.13) affecting the fields but not the coordinates $z^{M}$. The infinitesimal action variation under such a transformation on the field $F$ reads generically as

$$
\begin{aligned}
\underline{\delta}\left(S_{\Psi}+S_{\mathrm{B}}\right)= & \int d^{4} x\left\{-\left.\underline{\delta} F^{\alpha} \frac{\partial \mathcal{L}_{\mathrm{B}}}{\partial F^{\alpha}}\right|_{0}-\left.\underline{\delta} \bar{F}^{\alpha} \frac{\partial \mathcal{L}_{\mathrm{B}}}{\partial \bar{F}^{\alpha}}\right|_{0}+\left.\underline{\delta} F^{\alpha} \frac{\partial \mathcal{L}_{\mathrm{B}}}{\partial F^{\alpha}}\right|_{L}+\left.\underline{\delta} \bar{F}^{\alpha} \frac{\partial \mathcal{L}_{\mathrm{B}}}{\partial \bar{F}^{\alpha}}\right|_{L}\right\} \\
& +\int d^{4} x d y\left\{\underline{\delta} F^{\alpha} \frac{\partial \mathcal{L}_{\Psi}}{\partial F^{\alpha}}+\underline{\delta} \bar{F}^{\alpha} \frac{\partial \mathcal{L}_{\Psi}}{\partial \bar{F}^{\alpha}}+\underline{\delta}\left(\partial_{M} F^{\alpha}\right) \frac{\partial \mathcal{L}_{\Psi}}{\partial\left(\partial_{M} F^{\alpha}\right)}+\underline{\delta}\left(\partial_{M} \bar{F}^{\alpha}\right) \frac{\partial \mathcal{L}_{\Psi}}{\partial\left(\partial_{M} \bar{F}^{\alpha}\right)}\right\} .
\end{aligned}
$$

Now, we invoke the generic version of the EOM, $\frac{\partial \mathcal{L}_{\Psi}}{\partial F^{\alpha}}=\partial_{M} \frac{\partial \mathcal{L}_{\Psi}}{\partial\left(\partial_{M} F^{\alpha}\right)}$, as found in Eq. (3.3), ${ }^{52}$ not including the possible BBT contributions rather involved in the separate NBCs, as found in Eqs. (3.4)-(3.19) (without BBTs) and (3.29)-(3.33) (with BBTs). Using these EOM to rewrite the first two terms in the second line of Eq. (B1) and then grouping those with the last two terms to make global derivatives appear, we find

$$
\begin{aligned}
\underline{\delta}\left(S_{\Psi}+S_{\mathrm{B}}\right)= & \int d^{4} x\left\{-\left.\underline{\delta} F^{\alpha} \frac{\partial \mathcal{L}_{\mathrm{B}}}{\partial F^{\alpha}}\right|_{0}-\left.\underline{\delta} \bar{F}^{\alpha} \frac{\partial \mathcal{L}_{\mathrm{B}}}{\partial \bar{F}^{\alpha}}\right|_{0}+\left.\underline{\delta} F^{\alpha} \frac{\partial \mathcal{L}_{\mathrm{B}}}{\partial F^{\alpha}}\right|_{L}+\left.\underline{\delta} \bar{F}^{\alpha} \frac{\partial \mathcal{L}_{\mathrm{B}}}{\partial \bar{F}^{\alpha}}\right|_{L}\right\} \\
& +\int d^{4} x d y\left\{\partial_{M}\left(\underline{\delta} F^{\alpha} \frac{\partial \mathcal{L}_{\Psi}}{\partial\left(\partial_{M} F^{\alpha}\right)}\right)+\partial_{M}\left(\underline{\delta} \bar{F}^{\alpha} \frac{\partial \mathcal{L}_{\Psi}}{\partial\left(\partial_{M} \bar{F}^{\alpha}\right)}\right)\right\}
\end{aligned}
$$

The four terms in the first line (right-hand side) of this equation vanish since the infinitesimal field variations (3.14) lead, for instance, to

$$
-\left.\underline{\delta} Q^{\alpha} \frac{\partial \mathcal{L}_{\mathrm{B}}}{\partial Q^{\alpha}}\right|_{0}-\left.\underline{\delta} \bar{Q}^{\alpha} \frac{\partial \mathcal{L}_{\mathrm{B}}}{\partial \bar{Q}^{\alpha}}\right|_{0}=\left.\frac{1}{2} \bar{Q}(i \alpha Q)\right|_{0}+\left.\frac{1}{2}(-i \alpha \bar{Q}) Q\right|_{0}=0
$$

A similar cancellation, due to the symmetry of the model, arises for the last two terms at $y=L$ and the $D$ field contributions (relying on the $\alpha^{\prime}$ parameter).

The infinitesimal variation of the invariant Lagrangian from Eqs. (2.1) and (2.4) vanishes when integrated over the whole space $\left[\delta\left(S_{\psi}+S_{B}\right)=0\right]$ and even over any 5D domain $\Omega$, as the transformation affects the fields only. The first line (right-hand side) of Eq. (B2) vanishes as well for any integration volume $\Omega$ due to relations of type (B3) when $\Omega$ includes the boundaries $y=0, L$ and due to the absence of a Dirac peak in the integration domain in the other case. Therefore, mathematically, Eq. (B2) implies the vanishing of its second line for any integration region $\Omega$ and in turn (the fields being fixed by the geometrical model configuration over the whole interval) the local conservation relation for the 5D probability current of the field $F$,

$$
\begin{aligned}
\partial_{M} j_{F}^{M} & =0, \quad \text { for any } z^{M}, \quad \text { with } \\
j_{F}^{M} & =\underline{\delta} F^{\alpha} \frac{\partial \mathcal{L}_{\Psi}}{\partial\left(\partial_{M} F^{\alpha}\right)}+\underline{\delta} \bar{F}^{\alpha} \frac{\partial \mathcal{L}_{\Psi}}{\partial\left(\partial_{M} \bar{F}^{\alpha}\right)} .
\end{aligned}
$$

\footnotetext{
${ }^{52}$ Of course, similar EOM hold for the complex conjugate fields.
}

Note that an alternative instructive reading, based on the global derivatives of the second line in Eq. (B2) and an integration over a generic $5 \mathrm{D}$ domain $\Omega$, is that the second line vanishing leads to a cancellation of the sum over the differences of current components (each difference integrated over the complementary dimensions). This cancellation expresses the 5-current conservation over all directions (equality of the global ingoing and outgoing currents with respect to a given hypervolume $\Omega$ ) and is thus nothing else but a strictly equivalent, and less convenient, form of the conservation relation (B4): global versus local conservation of the full current $j_{F}^{M}$. For a consistency check, let us wonder what happens when the entire $5 \mathrm{D}$ domain is considered (i.e., $\Omega$ represents the whole 5D bulk). Then, the differences $j_{F}^{\mu}(+\infty, y)-j_{F}^{\mu}(-\infty, y)$ tend to zero-due to the vanishing of fields at infinite coordinates imposed by the vanishing boundary terms issued from the least action principle and independently to the wave function normalization conditions-so that one gets $\int d^{4} x\left[j_{F}^{4}\left(x^{\mu}, L\right)-j_{F}^{4}\left(x^{\mu}, 0\right)\right]=0$. This specific conservation property of the 5-current (or of the matter presence probability) must be compatible with the geometrical field configuration defining the model. The definition of the interval, $j_{F}^{4}\left(x^{\mu}, L\right)=j_{F}^{4}\left(x^{\mu}, 0\right)=0\left[\forall x^{\mu}\right]$ as 
in Eq. (3.15) for the present scenario, satisfies well this conservation property. It is obviously not the only way to respect the property. For example, within an orbifold scenario, the boundary point identification establishing the space periodicity, $y=0 \equiv L \Rightarrow$ $j_{F}^{4}\left(x^{\mu}, 0\right)=j_{F}^{4}\left(x^{\mu}, L\right)$, realizes as well the mentioned conservation pattern.
Let us now extend the demonstration of the Noether theorem to the presence of BBTs and boundary-localized Yukawa couplings by considering the free action (2.1) together with the BBTs (2.4) and the Yukawa terms (2.7). This whole action $S_{\Psi}+S_{\mathrm{B}}+S_{\mathrm{Y}}$ is invariant under the transformation (5.14). The infinitesimal action variation under this transformation reads as

$$
\begin{aligned}
\underline{\delta}\left(S_{\Psi}+S_{\mathrm{B}}+S_{\mathrm{Y}}\right)= & \sum_{F=Q_{L / R}, D_{L / R}} \int d^{4} x\left\{-\left.\underline{\delta} F^{\alpha} \frac{\partial \mathcal{L}_{\mathrm{Y}}}{\partial F^{\alpha}}\right|_{L}-\left.\underline{\delta} F^{\dagger \alpha} \frac{\partial \mathcal{L}_{\mathrm{Y}}}{\partial F^{\dagger \alpha}}\right|_{L}\right\} \\
& +\sum_{F=Q, D} \int d^{4} x\left\{-\left.\underline{\delta} F^{\alpha} \frac{\partial \mathcal{L}_{\mathrm{B}}}{\partial F^{\alpha}}\right|_{0}-\left.\underline{\delta} \bar{F}^{\alpha} \frac{\partial \mathcal{L}_{\mathrm{B}}}{\partial \bar{F}^{\alpha}}\right|_{0}+\left.\underline{\delta} F^{\alpha} \frac{\partial \mathcal{L}_{\mathrm{B}}}{\partial F^{\alpha}}\right|_{L}+\left.\underline{\delta} \bar{F}^{\alpha} \frac{\partial \mathcal{L}_{\mathrm{B}}}{\partial \bar{F}^{\alpha}}\right|_{L}\right\} \\
& +\sum_{F=Q, D} \int d^{4} x d y\left\{\underline{\delta} F^{\alpha} \frac{\partial \mathcal{L}_{\Psi}}{\partial F^{\alpha}}+\underline{\delta} \bar{F}^{\alpha} \frac{\partial \mathcal{L}_{\Psi}}{\partial \bar{F}^{\alpha}}+\underline{\delta}\left(\partial_{M} F^{\alpha}\right) \frac{\partial \mathcal{L}_{\Psi}}{\partial\left(\partial_{M} F^{\alpha}\right)}+\underline{\delta}\left(\partial_{M} \bar{F}^{\alpha}\right) \frac{\partial \mathcal{L}_{\Psi}}{\partial\left(\partial_{M} \bar{F}^{\alpha}\right)}\right\} .
\end{aligned}
$$

Invoking once more the EOM, $\frac{\partial \mathcal{L}_{\Psi}}{\partial F^{\alpha}}=\partial_{M} \frac{\partial \mathcal{L}_{\Psi}}{\partial\left(\partial_{M} F^{\alpha}\right.}$, including neither the possible BBT contributions nor the Yukawa terms (both rather entering the separate NBCs), we can rewrite the first two terms in the third line of Eq. (B5) and then group those with the last two terms to make global derivatives arise:

$$
\begin{aligned}
\underline{\delta}\left(S_{\Psi}+S_{\mathrm{B}}+S_{\mathrm{Y}}\right)= & \sum_{F=Q_{L / R}, D_{L / R}} \int d^{4} x\left\{-\left.\underline{\delta} F^{\alpha} \frac{\partial \mathcal{L}_{\mathrm{Y}}}{\partial F^{\alpha}}\right|_{L}-\left.\underline{\delta} F^{\dagger \alpha} \frac{\partial \mathcal{L}_{\mathrm{Y}}}{\partial F^{\dagger \alpha}}\right|_{L}\right\} \\
& +\sum_{F=Q, D} \int d^{4} x\left\{-\left.\underline{\delta} F^{\alpha} \frac{\partial \mathcal{L}_{\mathrm{B}}}{\partial F^{\alpha}}\right|_{0}-\left.\underline{\delta} \bar{F}^{\alpha} \frac{\partial \mathcal{L}_{\mathrm{B}}}{\partial \bar{F}^{\alpha}}\right|_{0}+\left.\underline{\delta} F^{\alpha} \frac{\partial \mathcal{L}_{\mathrm{B}}}{\partial F^{\alpha}}\right|_{L}+\left.\underline{\delta} \bar{F}^{\alpha} \frac{\partial \mathcal{L}_{\mathrm{B}}}{\partial \bar{F}^{\alpha}}\right|_{L}\right\} \\
& +\sum_{F=Q, D} \int d^{4} x d y\left\{\partial_{M}\left(\underline{\delta} F^{\alpha} \frac{\partial \mathcal{L}_{\Psi}}{\partial\left(\partial_{M} F^{\alpha}\right)}\right)+\partial_{M}\left(\underline{\delta} \bar{F}^{\alpha} \frac{\partial \mathcal{L}_{\Psi}}{\partial\left(\partial_{M} \bar{F}^{\alpha}\right)}\right)\right\} .
\end{aligned}
$$

Here, the four terms in the second line cancel each other since, for example, the infinitesimal field variations (5.15) lead to

$$
-\left.\underline{\delta} Q^{\alpha} \frac{\partial \mathcal{L}_{\mathrm{B}}}{\partial Q^{\alpha}}\right|_{0}-\left.\underline{\delta} \bar{Q}^{\alpha} \frac{\partial \mathcal{L}_{\mathrm{B}}}{\partial \bar{Q}^{\alpha}}\right|_{0}=\left.\frac{1}{2} \bar{Q}(i \alpha Q)\right|_{0}+\left.\frac{1}{2}(-i \alpha \bar{Q}) Q\right|_{0}=0,
$$

and the first line (right-hand side) vanishes as, for instance, the infinitesimal field variations of type (5.15) lead to

$$
\sum_{F=Q_{L / R}, D_{L / R}}\left[-\left.\underline{\delta} F^{\alpha} \frac{\partial\left(Y_{5} Q_{L}^{\dagger} H D_{R}\right)}{\partial F^{\alpha}}\right|_{L}-\left.\underline{\delta} F^{\dagger \alpha} \frac{\partial\left(Y_{5} Q_{L}^{\dagger} H D_{R}\right)}{\partial F^{\dagger \alpha}}\right|_{L}\right]=-\left.Y_{5} Q_{L}^{\dagger} H\left(i \alpha D_{R}\right)\right|_{L}-\left.Y_{5}\left(-i \alpha Q_{L}^{\dagger}\right) H D_{R}\right|_{L}=0 .
$$

Therefore, considerations on the vanishing infinitesimal variation (B6) over a generic $5 \mathrm{D}$ domain $\Omega$, similar as in the free case, lead to the local conservation relation for the 5D probability current,

$$
\partial_{M} j^{M}=0, \quad \text { for any } z^{M}, \quad \text { with } \quad j^{M}=\sum_{F=Q, D} \underline{\delta} F^{\alpha} \frac{\partial \mathcal{L}_{\Psi}}{\partial\left(\partial_{M} F^{\alpha}\right)}+\underline{\delta} \bar{F}^{\alpha} \frac{\partial \mathcal{L}_{\Psi}}{\partial\left(\partial_{M} \bar{F}^{\alpha}\right)} .
$$

\section{APPENDIX C: BOUNDARY CONDITIONS}

In this Appendix, we write down the global boundary condition derived from the initial variation of the action $S_{5 \mathrm{D}}^{\mathrm{m}}$ in Eq. (5.1):

$$
\left.\delta_{Q_{L}^{\dagger}} S\right|_{\mathrm{b}}+\left.\delta_{Q_{R}^{\ddagger}} S\right|_{\mathrm{b}}+\left.\delta_{D_{L}^{\dagger}} S\right|_{\mathrm{b}}+\left.\delta_{D_{R}^{\ddagger}} S\right|_{\mathrm{b}}+\left.\delta_{Q_{L}} S\right|_{\mathrm{b}}+\left.\delta_{Q_{R}} S\right|_{\mathrm{b}}+\left.\delta_{D_{L}} S\right|_{\mathrm{b}}+\left.\delta_{D_{R}} S\right|_{\mathrm{b}}=0, \quad \text { with, }
$$




$$
\begin{aligned}
& \left.\delta_{Q_{L}^{\dagger}} S_{5 \mathrm{D}}^{\mathrm{m}} \ni \delta_{Q_{L}^{\dagger}} S\right|_{\mathrm{b}}=\left.\int d^{4} x\left[\delta Q_{L}^{\dagger}\left(\frac{1}{2} Q_{R}-X D_{R}\right)\right]\right|_{L}-\left.\int d^{4} x\left(\delta Q_{L}^{\dagger} Q_{R}\right)\right|_{0}, \\
& \left.\delta_{Q_{R}^{\dagger}} S_{5 \mathrm{D}}^{\mathrm{m}} \ni \delta_{Q_{R}^{\dagger}} S\right|_{\mathrm{b}}=\left.\int d^{4} x\left[-\delta Q_{R}^{\dagger}\left(\frac{1}{2} Q_{L}+X^{\prime} D_{L}\right)\right]\right|_{L}, \\
& \left.\delta_{D_{L}^{\dagger}} S_{5 \mathrm{D}}^{\mathrm{m}} \ni \delta_{D_{L}^{\dagger}} S\right|_{\mathrm{b}}=\left.\int d^{4} x\left[\delta D_{L}^{\dagger}\left(\frac{1}{2} D_{R}-X^{\prime \star} Q_{R}\right)\right]\right|_{L}, \\
& \left.\delta_{D_{R}^{\dagger}} S_{5 \mathrm{D}}^{\mathrm{m}} \ni \delta_{D_{R}^{\dagger}} S\right|_{\mathrm{b}}=\left.\int d^{4} x\left[-\delta D_{R}^{\dagger}\left(\frac{1}{2} D_{L}+X^{\star} Q_{L}\right)\right]\right|_{L}+\left.\int d^{4} x\left(\delta D_{R}^{\dagger} D_{L}\right)\right|_{0}, \\
& \left.\delta_{Q_{L}} S_{5 \mathrm{D}}^{\mathrm{m}} \ni \delta_{Q_{L}} S\right|_{\mathrm{b}}=\left.\int d^{4} x\left[\left(\frac{1}{2} Q_{R}^{\dagger}-X^{\star} D_{R}^{\dagger}\right) \delta Q_{L}\right]\right|_{L}-\left.\int d^{4} x\left(Q_{R}^{\dagger} \delta Q_{L}\right)\right|_{0}, \\
& \left.\delta_{Q_{R}} S_{5 \mathrm{D}}^{\mathrm{m}} \ni \delta_{Q_{R}} S\right|_{\mathrm{b}}=\left.\int d^{4} x\left[-\left(\frac{1}{2} Q_{L}^{\dagger}+X^{\prime \star} D_{L}^{\dagger}\right) \delta Q_{R}\right]\right|_{L}, \\
& \left.\delta_{D_{L}} S_{5 \mathrm{D}}^{\mathrm{m}} \ni \delta_{D_{L}} S\right|_{\mathrm{b}}=\left.\int d^{4} x\left[\left(\frac{1}{2} D_{R}^{\dagger}-X^{\prime} Q_{R}^{\dagger}\right) \delta D_{L}\right]\right|_{L}, \\
& \left.\delta_{D_{R}} S_{5 \mathrm{D}}^{\mathrm{m}} \ni \delta_{D_{R}} S\right|_{\mathrm{b}}=\left.\int d^{4} x\left[-\left(\frac{1}{2} D_{L}^{\dagger}+X Q_{L}^{\dagger}\right) \delta D_{R}\right]\right|_{L}+\left.\int d^{4} x\left(D_{L}^{\dagger} \delta D_{R}\right)\right|_{0}
\end{aligned}
$$

[1] L. Randall and R. Sundrum, A Large Mass Hierarchy from a Small Extra Dimension, Phys. Rev. Lett. 83, 3370 (1999).

[2] S. Chang, J. Hisano, H. Nakano, N. Okada, and M. Yamaguchi, Bulk Standard Model in the Randall-Sundrum background, Phys. Rev. D 62, 084025 (2000).

[3] T. Gherghetta and A. Pomarol, Bulk fields and supersymmetry in a slice of AdS, Nucl. Phys. B586, 141 (2000).

[4] S. J. Huber and Q. Shafi, Neutrino oscillations and rare processes in models with a small extra dimension, Phys. Lett. B 512, 365 (2001).

[5] S. Casagrande, F. Goertz, U. Haisch, M. Neubert, and T. Pfoh, Flavor physics in the Randall-Sundrum model: I. Theoretical setup and electroweak precision tests, J. High Energy Phys. 10 (2008) 094.

[6] S. Chang, C. S. Kim, and M. Yamaguchi, Hierarchical mass structure of fermions in warped extra dimension, Phys. Rev. D 73, 033002 (2006).

[7] G. Moreau and J. I. Silva-Marcos, Neutrinos in warped extra dimensions, J. High Energy Phys. 01 (2006) 048.

[8] G. Moreau and J. I. Silva-Marcos, Flavor physics of the RS model with KK masses reachable at LHC, J. High Energy Phys. 03 (2006) 090.

[9] C. Bouchart and G. Moreau, The precision electroweak data in warped extra-dimension models, Nucl. Phys. B810, 66 (2009).

[10] F. Englert and R. Brout, Broken Symmetry and the Mass of Gauge Vector Mesons, Phys. Rev. Lett. 13, 321 (1964).

[11] P. W. Higgs, Broken symmetries, massless particles and gauge fields, Phys. Lett. 12, 132 (1964).

[12] K. R. Dienes, E. Dudas, and T. Gherghetta, Neutrino oscillations without neutrino masses or heavy mass scales:
A higher dimensional seesaw mechanism, Nucl. Phys. B557, 25 (1999).

[13] A. Abada, P. Dey, and G. Moreau, Neutrinos in flat extra dimension: Towards a realistic scenario, J. High Energy Phys. 09 (2007) 006.

[14] Y. Grossman and M. Neubert, Neutrino masses and mixings in nonfactorizable geometry, Phys. Lett. B 474, 361 (2000).

[15] S. J. Huber and Q. Shafi, Majorana neutrinos in a warped 5-D standard model, Phys. Lett. B 544, 295 (2002).

[16] G. Moreau, Realistic neutrino masses from multi-brane extensions of the Randall-Sundrum model?, Eur. Phys. J. C 40, 539 (2005).

[17] J. M. Frere, G. Moreau, and E. Nezri, Neutrino mass patterns within the seesaw model from multilocalization along extra dimensions, Phys. Rev. D 69, 033003 (2004).

[18] J. Polchinski, Tasi lectures on D-branes, in Fields, strings and duality, Proceedings, Summer School, Theoretical Advanced Study Institute in Elementary Particle Physics, TASI'96, Boulder, USA (1996), pp. 293-356.

[19] C. P. Bachas, Lectures on D-branes, in Duality and supersymmetric theories, Proceedings, Easter School, Newton Institute, Euroconference, Cambridge, UK, 1997 (1998), pp. 414-473.

[20] O. Aharony, S. S. Gubser, J. M. Maldacena, H. Ooguri, and Y. Oz, Large $\mathrm{N}$ field theories, string theory and gravity, Phys. Rep. 323, 183 (2000).

[21] M. J. Duff, Supermembranes, in 26th British Universities Summer School in Theoretical Elementary Particle Physics (BUSSTEPP 1996) Swansea, Wales (1996). 
[22] L. J. Hall, Y. Nomura, and D. Tucker-Smith, Gauge Higgs unification in higher dimensions, Nucl. Phys. B639, 307 (2002).

[23] M. N. Smolyakov, Unremovable divergencies in fourdimensional electrodynamics localized on a domain wall, Phys. Rev. D 85, 045036 (2012).

[24] S. Fichet, Braneworld Effective field theories-holography, consistency and conformal effects, arXiv:1912.12316.

[25] L. F. F. Freitas, G. Alencar, and R. R. Landim, Consistency conditions for fields localization on braneworlds, arXiv: 2001.01267.

[26] J. A. Bagger, F. Feruglio, and F. Zwirner, Generalized Symmetry Breaking on Orbifolds, Phys. Rev. Lett. 88, 101601 (2002).

[27] C. Csaki, C. Grojean, J. Hubisz, Y. Shirman, and J. Terning, Fermions on an interval: Quark and lepton masses without a Higgs, Phys. Rev. D 70, 015012 (2004).

[28] A. Azatov, M. Toharia, and L. Zhu, Higgs mediated flavor changing neutral currents in warped extra dimensions, Phys. Rev. D 80, 035016 (2009).

[29] F. Goertz and T. Pfoh, On the perturbative approach in the Randall-Sundrum model, J. High Energy Phys. 10 (2008) 035 .

[30] S. Casagrande, F. Goertz, U. Haisch, M. Neubert, and T. Pfoh, The custodial Randall-Sundrum model: From precision tests to Higgs physics, J. High Energy Phys. 09 (2010) 014.

[31] M. Carena, S. Casagrande, F. Goertz, U. Haisch, and M. Neubert, Higgs production in a warped extra dimension, J. High Energy Phys. 08 (2012) 156.

[32] R. Malm, M. Neubert, K. Novotny, and C. Schmell, 5D perspective on Higgs production at the boundary of a warped extra dimension, J. High Energy Phys. 01 (2014) 173.

[33] J. Hahn, C. Hörner, R. Malm, M. Neubert, K. Novotny, and C. Schmell, Higgs decay into two photons at the boundary of a warped extra dimension, Eur. Phys. J. C 74, 2857 (2014).

[34] A. Azatov, M. Toharia, and L. Zhu, Higgs production from gluon fusion in warped extra dimensions, Phys. Rev. D 82, 056004 (2010).

[35] R. Barceló, S. Mitra, and G. Moreau, On a boundarylocalized Higgs boson in 5D theories, Eur. Phys. J. C 75, 527 (2015).

[36] R. Courant and D. Hilbert, Methods of Mathematical Physics, Vol. 1 (Interscience Publishers, New York, 1953).

[37] L. Schwartz, Mathematics for the Physical Sciences (Hermann, Paris, 1966).

[38] L. Schwartz, Théorie des Distributions (Hermann, Paris, 1966).

[39] K. Agashe, A. Delgado, M. J. May, and R. Sundrum, RS1, Custodial isospin and precision tests, J. High Energy Phys. 08 (2003) 050.

[40] M. Henningson and K. Sfetsos, Spinors and the AdS/CFT correspondence, Phys. Lett. B 431, 63 (1998).

[41] W. Mueck and K. S. Viswanathan, Conformal field theory correlators from classical field theory on anti-de Sitter space. 2. Vector and spinor fields, Phys. Rev. D 58, 106006 (1998).
[42] R. Contino and A. Pomarol, Holography for fermions, J. High Energy Phys. 11 (2004) 058.

[43] M. Henneaux, Boundary terms in the AdS/CFT correspondence for spinor fields, in Mathematical Methods in Modern Theoretical Physics. Proceedings, International Meeting, School and Workshop, ISPM'98, Tbilisi, Georgia (1998), pp. 161-170.

[44] G. E. Arutyunov and S. A. Frolov, On the origin of supergravity boundary terms in the AdS/CFT correspondence, Nucl. Phys. B544, 576 (1999).

[45] G. W. Gibbons and S. W. Hawking, Action integrals and partition functions in quantum gravity, Phys. Rev. D 15, 2752 (1977).

[46] H. A. Chamblin and H. S. Reall, Dynamic dilatonic domain walls, Nucl. Phys. B562, 133 (1999).

[47] Z. Lalak and R. Matyszkiewicz, Boundary terms in brane worlds, J. High Energy Phys. 11 (2001) 027.

[48] M. Carena, J. D. Lykken, and M. Park, Interval approach to braneworld gravity, Phys. Rev. D 72, 084017 (2005).

[49] Study under progess.

[50] H.-C. Cheng, Introduction to extra dimensions, in Physics of the Large and the Small, TASI 09, Proceedings of the Theoretical Advanced Study Institute in Elementary Particle Physics, Boulder, Colorado, USA, 2009 (2011), pp. 125-162.

[51] C. Csaki, J. Hubisz, and P. Meade, TASI lectures on electroweak symmetry breaking from extra dimensions, in Physics in $D \geq 4$, Proceedings, Theoretical Advanced Study Institute in Elementary Particle Physics, TASI 2004, Boulder, USA, 2004 (2005), pp. 703-776.

[52] T. Gherghetta, Les Houches lectures on warped models and holography, in Particle Physics beyond the Standard Model. Proceedings, Summer School on Theoretical Physics, 84th Session, Les Houches, France, 2005 (2006), pp. 263-311.

[53] C. Grojean, New approaches to electroweak symmetry breaking, Phys. Usp. 50, 1 (2007).

[54] B. A. Dobrescu, Particle physics in extra dimensions, in Proceedings of Theoretical Advanced Study Institute in Elementary Particle Physics on The dawn of the LHC Era (TASI 2008): Boulder, USA, 2008 (2010), pp. 495-523.

[55] T. Gherghetta, A holographic view of beyond the Standard Model physics, in Physics of the Large and the Small, TASI 09, Proceedings of the Theoretical Advanced Study Institute in Elementary Particle Physics, Boulder, Colorado, USA, 2009 (2011), pp. 165-232.

[56] E. Ponton, TASI 2011: Four lectures on TeV scale extra dimensions, in The Dark Secrets of the Terascale: Proceedings, TASI 2011, Boulder, Colorado, USA, 2011 (2013), pp. 283-374.

[57] S. Raychaudhuri and K. Sridhar, Particle Physics of Brane Worlds and Extra Dimensions, Cambridge Monographs on Mathematical Physics (Cambridge University Press, Cambridge, England, 2016).

[58] M. Giaquinta and S. Hildebrandt, Calculus of Variations I: The Lagrangian Formalism. Grundlehren der Mathematischen Wissenschaften (Springer, New York, 2004).

[59] M. Burgess, Classical Covariant Fields, Cambridge Monographs on Mathematical Physics (Cambridge University Press, Cambridge, England, 2005). 
[60] S. J. Huber and Q. Shafi, Higgs mechanism and bulk gauge boson masses in the Randall-Sundrum model, Phys. Rev. D 63, 045010 (2001).

[61] F. del Aguila and J. Santiago, Universality limits on bulk fermions, Phys. Lett. B 493, 175 (2000).

[62] J. L. Hewett, F. J. Petriello, and T. G. Rizzo, Precision measurements and fermion geography in the Randall-Sundrum model revisited, J. High Energy Phys. 09 (2002) 030.

[63] S. J. Huber, Flavor violation and warped geometry, Nucl. Phys. B666, 269 (2003).

[64] K. Agashe, A. E. Blechman, and F. Petriello, Probing the Randall-Sundrum geometric origin of flavor with lepton flavor violation, Phys. Rev. D 74, 053011 (2006).

[65] K. Agashe, G. Perez, and A. Soni, Collider signals of top quark flavor violation from a warped extra dimension, Phys. Rev. D 75, 015002 (2007).

[66] A. Djouadi and G. Moreau, Higgs production at the LHC in warped extra-dimensional models, Phys. Lett. B 660, 67 (2008).

[67] C. Bouchart and G. Moreau, Higgs boson phenomenology and vacuum expectation value shift in the Randall-Sundrum scenario, Phys. Rev. D 80, 095022 (2009).
[68] P. A. M. Dirac, The physical interpretation of the quantum dynamics, Proc. R. Soc. A 113, 621 (1927).

[69] C. Bouchart, A. Knochel, and G. Moreau, Discriminating 4D supersymmetry from its 5D warped version, Phys. Rev. D 84, 015016 (2011).

[70] F. Burk, Lebesgue Measure and Integration: An Introduction (Wiley, New York, 1998).

[71] S. J. Huber and Q. Shafi, Fermion masses, mixings and proton decay in a Randall-Sundrum model, Phys. Lett. B 498, 256 (2001).

[72] S. J. Huber and Q. Shafi, Seesaw mechanism in warped geometry, Phys. Lett. B 583, 293 (2004).

[73] F. Ledroit, G. Moreau, and J. Morel, Probing RS scenarios of flavour at LHC via leptonic channels, J. High Energy Phys. 09 (2007) 071.

[74] S. Gopalakrishna, T. Mandal, S. Mitra, and G. Moreau, LHC signatures of warped-space vectorlike quarks, J. High Energy Phys. 08 (2014) 079.

[75] M. D. Schwartz, Quantum Field Theory and the Standard Model (Cambridge University Press, Cambridge, England, 2014). 